\title{
SPRINGER CORRESPONDENCE, HYPERELLIPTIC CURVES, AND COHOMOLOGY OF FANO VARIETIES
}

\author{
TSAO-HSIEN CHEN, KARI VILONEN, AND TING XUE
}

\begin{abstract}
In CVX3, we have established a Springer theory for the symmetric pair $(\mathrm{SL}(N), \mathrm{SO}(N))$. In this setting we obtain representations of (the Tits extension) of the braid group rather than just Weyl group representations. These representations arise from cohomology of families of certain (Hessenberg) varieties. In this paper we determine the Springer correspondence explicitly for IC sheaves supported on order 2 nilpotent orbits. In this process we encounter universal families of hyperelliptic curves. As an application we calculate the cohomolgy of Fano varieties of $k$-planes in the smooth intersection of two quadrics in an even dimensional projective space.
\end{abstract}

\section{Contents}

1. Introduction 1

2. Springer correspondence 3

3. Bijection of sets 6

4. Proof of the main theorems 10

5. Stalks of the IC sheaves 19

6. Cohomology of Fano varieties 24

\begin{tabular}{ll} 
References & 29 \\
\hline
\end{tabular}

\section{INTRODUCTION}

In CVX3 we have established a Springer correspondence for the symmetric pairs $(\mathrm{SL}(N), \mathrm{SO}(N))$. More precisely, let $G=\mathrm{SL}(N), \mathfrak{g}=\operatorname{Lie} G=\mathfrak{s l}(N)$, and $\theta$ an involution of $G$ such that $K=G^{\theta}=\mathrm{SO}(N)$. The involution $\theta$ gives rise to a decomposition $\mathfrak{g}=\mathfrak{g}_{0} \oplus \mathfrak{g}_{1}$, where $\left.d \theta\right|_{\mathfrak{g}_{i}}=(-1)^{i}, i=0,1$. Let $\mathcal{N}$ be the nilpotent cone of $\mathfrak{g}$ and let $\mathcal{N}_{1}=\mathcal{N} \cap \mathfrak{g}_{1}$. In CVX3] we determine the set of Fourier transforms of all irreducible $K$ equivariant perverse sheaves on $\mathcal{N}_{1}$, i.e., the set of Fourier transforms $\mathfrak{F}(\operatorname{IC}(\mathcal{O}, \mathcal{E})$ ), where

Tsao-Hsien Chen was partially supported by NSF grant DMS-1702337 and DMS-2001257.

Kari Vilonen was supported in part by the ARC grants DP150103525 and DP180101445, the Academy of Finland, the Humboldt Foundation, the Simons Foundation, and the NSF grant DMS-1402928.

Ting Xue was supported in part by the ARC grants DP150103525 and DE160100975 and the Academy of Finland. 
$\mathcal{O}$ is a nilpotent $K$-orbit in $\mathcal{N}_{1}, \mathcal{E}$ is an irreducible $K$-equivariant local system on $\mathcal{O}$, and $\mathfrak{F}: \operatorname{Perv}_{K}\left(\mathfrak{g}_{1}\right) \rightarrow \operatorname{Perv}_{K}\left(\mathfrak{g}_{1}\right)$ is the Fourier transform functor (where we have identified $\mathfrak{g}_{1}$ with $\mathfrak{g}_{1}^{*}$ via the Killing form). Several things remain to be done. For example, we do not know the Fourier transform of a specific $\operatorname{IC}(\mathcal{O}, \mathcal{E})$ in general, although we have some partial conjectures [CVX3, Conjecture 7.1]. Also, very little is known about the geometric properties of the Fourier transforms $\mathfrak{F}(\mathrm{IC}(\mathcal{O}, \mathcal{E})$ ) (i.e. the character sheaves) and the work in loc. cit. suggests that the geometric properties of $\mathfrak{F}(\mathrm{IC}(\mathcal{O}, \mathcal{E}))$ are very different from those in the group case.

In this paper we explore the geometry of the Fourier transforms $\mathfrak{F}(\mathrm{IC}(\mathcal{O}, \mathcal{E}))$ in the simplest nontrivial case, that is, in the case when $\mathcal{O}$ is an order 2 nilpotent orbit 1 . We show that those Fourier transforms involve cohomology of a beautiful family of non-rational varieties and we use the geometry of those varieties to determine $\mathfrak{F}(\operatorname{IC}(\mathcal{O}, \mathcal{E}))$ for all order 2 nilpotent orbits $\mathcal{O}$. As an application, we compute the cohomology of Fano varieties of $k$-planes in the smooth intersection of two quadrics in an even dimensional projective space. When the ambient projective space is odd dimensional, the cohomology of such Fano varieties has been computed in CVX2 making use of the Springer correspondence in the case of $N$ even.

Let us assume that $N=2 n+1$. We show that for trivial local systems on order 2 nilpotent orbits the Fourier transforms are IC sheaves arising from representations of the Tits group $A[2] \rtimes S_{N}$, which do not factor through $S_{N}$; see Theorem 2.2. Here $A[2]$ is the set of order 2 elements in a fixed maximal $\theta$-split torus $A$ of $G$. In the case of non-trivial local systems the Fourier transforms are IC sheaves arising from representations of the braid group $B_{N}$ on the cohomology of a universal family of hyperelliptic curves; see Theorem 2.3.

As an application we obtain an explicit formula for the cohomology of the Fano varieties $\mathrm{Fano}_{i}^{2 n}$ of $i$-planes in the smooth complete intersection of two quadrics in an even dimensional projective space $\mathbb{P}^{2 n}$. This is an example of the following general strategy (see also [CVX2]). Consider pairs of maps $(\pi, \check{\pi})$ and diagrams as follows:

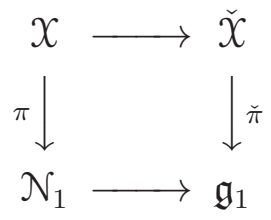

where $\mathcal{X}$ and $\check{X}$ are certain families of Hessenberg varieties, see [CVX1]. In particular, $X$ and $\check{X}$ are orthogonal vector bundles over a partial flag manifold $\mathcal{P}$ of $K$ in the trivial bundle $\mathcal{P} \times \mathfrak{g}_{1}$ so that, by functoriality of the Fourier transform, we have

$$
\mathfrak{F}\left(\pi_{*} \mathbb{C}_{x}\right) \cong \check{\pi}_{*} \mathbb{C}_{\check{x}} \quad \text { (up to shift). }
$$

In general the fibers of $\check{\pi}$ are more complicated than those of $\pi$. We make use of the following strategy:

The computation of the cohomology of the general fiber of $\check{x}$ can be reduced, via the Fourier transform, to the analysis of the boundary family $\mathcal{X}$.

\footnotetext{
${ }^{1} \mathrm{~A}$ nonzero nilpotent orbit $\mathcal{O}$ is called of order 2 if $x^{2}=0$ for all $x \in \mathcal{O}$.
} 
For this to work, we need to know the Fourier transforms of the IC sheaves that occur in the decomposition of $\pi_{*} \mathbb{C}_{x}$. In our setting we observe that the Fano varieties Fano $_{i}^{2 n}$ appear as general fibers of a certain families of Hessenberg varieties $\check{X}$ and by applying the principle above we obtain the following theorem. Let us write

$$
g_{k, m}(q)=\frac{\prod_{l=m-k+1}^{m}\left(1-q^{l}\right)}{\prod_{l=1}^{k}\left(1-q^{l}\right)}
$$

for the Poincare polynomial of the Grassmannian of $k$-dimensional subspaces in $\mathbb{C}^{m}$. Then:

Theorem 1.1 (Theorem 6.1). We have

$$
\begin{gathered}
H^{2 k+1}\left(\mathrm{Fano}_{i-1}^{2 n}, \mathbb{C}\right)=0, \\
H^{2 k}\left(\mathrm{Fano}_{i-1}^{2 n}, \mathbb{C}\right) \cong \bigoplus_{j=0}^{i} L_{j}^{\oplus M_{i}(k, j)},
\end{gathered}
$$

where $M_{i}(k, j)$ is the coefficient of $q^{k-j(n-i)}$ in $g_{i-j, 2 n-i-j}(q)$ and the $L_{j}$ are vector spaces of dimension $\left(\begin{array}{c}2 n+1 \\ j\end{array}\right)$.

Remark 1.2. It follows from the proof that there are natural actions of the braid group on the cohomology $H^{2 k}\left(\mathrm{Fano}_{i-1}^{2 n}, \mathbb{C}\right)$ and the vector space $L_{j}$ and the isomorphism in the theorem is compatible with those actions.

Remark 1.3. The Fano varieties $\mathrm{Fano}_{i}^{2 n}$ have a concrete interpretation as moduli space of parabolic vector bundles (with extra structure) on $\mathbb{P}^{1}$ (see [C]). Thus the theorem above provides a method to compute the cohomology of those moduli spaces.

To prove our main theorems we establish and make use of an interesting isomorphism of the cohomology of the stalks of IC sheaves attached to nilpotent orbits of order 2 and the cohomology of stalks of IC sheaves attached to nilpotent orbits in the symplectic Lie algebra $\mathfrak{s p}(2 n)$ in the classical case. This is done in $\$ 5$. The proofs of the results in this section are independent of the other sections and the results are used in $\$ 4$ and in $\$ 6$,

Acknowledgements. We thank Carl Mautner for useful discussions at the beginning of the project. We also thank the Max Planck Institute for Mathematics in Bonn and the Mathematical Sciences Research Institute in Berkeley for support, hospitality, and nice research environment. Furthermore KV and TX thank the Research Institute for Mathematical Sciences in Kyoto for support, hospitality, and nice research environment. We also thank Cheng-Chiang Tsai, Zhiwei Yun and Xinwen Zhu for their interest in this work. In addition, TX would like to thank George Lusztig for suggesting her to study the topics in this paper and for helpful discussions about fake degrees. The authors would also like to thank the anonymous referees for helpful comments. Finally, special thanks are due to Dennis Stanton for suppling a combinatorial argument used in section 6 .

\section{SPRINGER CORRESPONDENCE}

In this section we state our main results on Fourier transforms of IC complexes supported on nilpotent orbits of order two. 
2.1. Notations. Let $G=\operatorname{SL}(N, \mathbb{C})$ and $\theta: G \rightarrow G$ an involution such that $K=G^{\theta}=$ $\mathrm{SO}(N, \mathbb{C})$. We also write $(G, K)=(\mathrm{SL}(V), \mathrm{SO}(V, Q))$, where $\operatorname{dim} V=N$. We think of $Q$ concretely as a non-degenerate quadratic form on $V$ and we write $\langle,\rangle_{Q}$ for the non-degenerate bilinear form on $V$ associated to $Q$. The involution $\theta$ induces a grading on $\mathfrak{g}=$ Lie $G$, i.e. $\mathfrak{g}=\mathfrak{g}_{0} \oplus \mathfrak{g}_{1}$, where $\left.d \theta\right|_{\mathfrak{g}_{i}}=(-1)^{i}$. If we diagonalize $Q$ then the Cartan involution $\theta$ is given by $g \mapsto\left(g^{t}\right)^{-1}$ and $\mathfrak{g}_{1}$ consists of symmetric matrices.

We write $A$ for a maximal $\theta$-split torus of $G$, where $\theta$-split means that $\theta(t)=t^{-1}$ for $t \in A$. The pair $(G, K)$ is a split pair, i.e., $A$ is a maximal torus of $G$. We write $\mathfrak{g}^{r s}$ for the regular semi-simple elements in $\mathfrak{g}$ and we let $\mathfrak{g}_{1}^{r s}=\mathfrak{g}_{1} \cap \mathfrak{g}^{r s}$. Furthermore, let $\mathfrak{a}=$ Lie $A$ and $\mathfrak{a}^{r s}=\mathfrak{a} \cap \mathfrak{g}^{r s}$. Then $\mathfrak{a}$ is a maximal abelian subspace in $\mathfrak{g}_{1}$.

Let us write $\mathcal{N}$ for the nilpotent cone of $\mathfrak{g}$ and we let $\mathcal{N}_{1}=\mathcal{N} \cap \mathfrak{g}_{1}$ stand for the nilpotent cone of $\mathfrak{g}_{1}$. Assume that $N=2 n+1$. The $K$-orbits on $\mathcal{N}_{1}$ are parametrized by partitions $\lambda$ of $2 n+1$ and we write $\mathcal{O}_{\lambda}$ for the $K$-orbit corresponding to $\lambda$ (see [S]). For $i \in[0, n]$, let $2^{i} 1^{2 n+1-2 i}$ denote the partition where the part 2 has multiplicity $i$, and the part 1 has multiplicity $2 n+1-2 i$. The order 2 nilpotent orbits are $\mathcal{O}_{2^{i} 1^{2 n+1-2 i}}, i \in[1, n]$. For $x \in \mathcal{O}_{2^{i} 1^{2 n+1-2 i}}$, $i \in[1, n]$, we have that $A_{K}(x):=Z_{K}(x) / Z_{K}(x)^{0}=\mathbb{Z} / 2 \mathbb{Z}$. Thus each orbit $\mathcal{O}_{2^{i} 1^{2 n+1-2 i}}$ carries a unique non-trivial irreducible $K$-equivariant local system, $i \in[1, n]$; we denote it by $\mathcal{E}_{i}$.

By [CVX3, Corollary 4.9], we know that both $\mathfrak{F}\left(\operatorname{IC}\left(\mathcal{O}_{2^{i} 1^{2 n+1-2 i}}, \mathbb{C}\right)\right)$ and $\mathfrak{F}\left(\operatorname{IC}\left(\mathcal{O}_{2^{i} 1^{2 n+1-2 i}}, \mathcal{E}_{i}\right)\right)$ are of the form $\mathrm{IC}\left(\mathfrak{g}_{1}^{r s},-\right)$, where - is an irreducible $K$-equivariant local system on $\mathfrak{g}_{1}^{r s}$ corresponding to an irreducible representation of $\pi_{1}^{K}\left(\mathfrak{g}_{1}^{r s}\right)=\pi_{1}^{K}\left(\mathfrak{g}_{1}^{r s}, a\right), a \in \mathfrak{a}^{r s}$, the equivariant fundamental group.

2.2. The equivariant fundamental group. Let us describe the equivariant fundamental group $\pi_{1}^{K}\left(\mathfrak{g}_{1}^{r s}, a\right)$ concretely. In this subsection $N$ is arbitrary.

Let $\mathrm{W}_{1}=N_{K}(A) / Z_{K}(A)$ be the 'baby Weyl group'. Recall that we have

Lemma $2.1([\mathrm{KR}])$. The natural inclusion $\mathfrak{a} \hookrightarrow \mathfrak{g}_{1}$ induces an isomorphism $k[\mathfrak{a}]^{\mathrm{W}_{1}}=k\left[\mathfrak{g}_{1}\right]^{K}$. We have the relative Chevalley map $f: \mathfrak{g}_{1} \rightarrow \mathfrak{g}_{1} / / K \simeq \mathfrak{a} / \mathrm{W}_{1}:=\mathfrak{c}$.

Let $W$ be the Weyl group of $G$. In our setting, we have $W_{1}=W=S_{N}$. Let us write $c \in \mathfrak{c}^{r s}$ for the image of $a$ under $\mathfrak{a}^{r s} \rightarrow \mathfrak{c}^{r s}=\mathfrak{a}^{r s} / W$. Now, $B_{W}:=\pi_{1}\left(\mathfrak{c}^{r s}, c\right)=B_{N}$ is the classical braid group. Then the relative Chevalley map $f: \mathfrak{g}_{1} \rightarrow \mathfrak{c}$ gives rise to the following commutative diagram with exact rows:

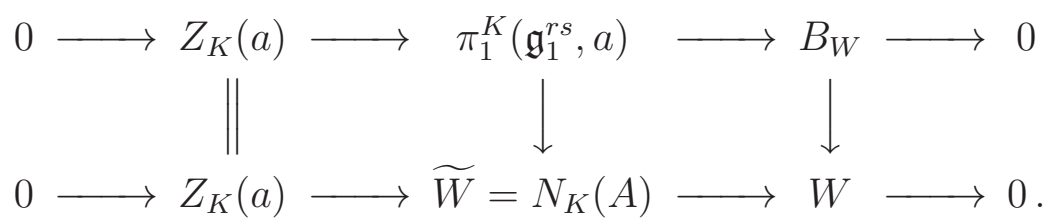

Note that $\widetilde{W}$ is the Tits group. The group $B_{W}$ acts on $Z_{K}(a)$ through the quotient $B_{W} \rightarrow W$. We have that $Z_{K}(a) \simeq A[2]$, the group of order 2 elements in the split torus $A$. Choosing a section we can split the short exact sequence in the first row and obtain

$$
\pi_{1}^{K}\left(\mathfrak{g}_{1}^{r s}, a\right) \simeq A[2] \rtimes B_{W} .
$$


However, note that the second row in the diagram is not split in general. In the case when $N$ is odd the lower exact sequence also splits and we have

$$
\widetilde{W}=N_{K}(A) \simeq A[2] \rtimes S_{N} .
$$

For the rest of the paper, we will assume $N$ is odd.

2.3. The local systems $\mathcal{L}_{i}$ and $\mathcal{F}_{i}$ and the statements of theorems. The local systems on $\mathfrak{g}_{1}^{r s}$ which are obtained from the Fourier transforms of $\operatorname{IC}\left(\mathcal{O}_{2^{i} 1^{2 n-2 i+1}}, \mathbb{C}\right)$ turn out to be representations of the Tits group $\widetilde{W}$. They are the irreducible representations which occur in $\mathbb{C}[A[2]]$ viewed as a representation of $\widetilde{W}$. First, let us write $L_{\chi}$ for the representation of $A[2]$ associated to the character $\chi \in A[2]^{\vee}$ and so we have

$$
\mathbb{C}[A[2]]=\bigoplus_{\chi \in A[2]^{\vee}} L_{\chi}
$$

The space $A[2]^{\vee}$ breaks into orbits $\left\{\Lambda_{i}\right\}_{i=0, \ldots, n}$ under the action of $W=S_{N}$, where we have numbered the orbits so that $\left|\Lambda_{i}\right|=\left(\begin{array}{c}2 n+1 \\ i\end{array}\right)$. Note that here it is crucial that $N=2 n+1$ is odd. Concretely, the $\Lambda_{i}$ consists of characters that attain the value $-1 \in \mathbb{G}_{m}$ precisely $i$ times if $i$ is even and $2 n+1-i$ times if $i$ is odd 2 . Thus, for each $i=0, \ldots, n$ we obtain an irreducible representation of $\widetilde{W}$ as follows

$$
L_{i}=\bigoplus_{\chi \in \Lambda_{i}} L_{\chi}
$$

We write $\mathcal{L}_{i}$ for the irreducible $K$-equivariant local system on $\mathfrak{g}_{1}^{\text {rs }}$ corresponding to the representation $L_{i}$ via the map $\pi_{1}^{K}\left(\mathfrak{g}_{1}^{r s}, a\right) \rightarrow \widetilde{W}$.

Theorem 2.2. We have $\mathfrak{F}\left(\mathrm{IC}\left(\mathcal{O}_{2^{i} 1^{2 n-2 i+1}}, \mathbb{C}\right)\right)=\operatorname{IC}\left(\mathfrak{g}_{1}, \mathcal{L}_{i}\right), i=0, \ldots, n$.

We will now define irreducible $K$-equivariant local systems $\mathcal{F}_{i}$ on $\mathfrak{g}_{1}^{\text {rs }}$ which are obtained from the Fourier transforms of $\operatorname{IC}\left(\mathcal{O}_{2^{i} 1^{2 n-2 i+1}}, \mathcal{E}_{i}\right)$ 's. These local systems arise as representations of $\pi_{1}\left(\mathfrak{c}^{r s}, a\right)=B_{N}$. To this end we consider the following universal family $C$ of hyperelliptic curves of genus $n$ :

To each $a=\left(a_{1}, \ldots, a_{2 n+1}\right) \in \mathfrak{a}^{r s}$ we associate the hyperelliptic

curve $C_{a}$ which ramifies at $\left\{a_{1}, \ldots, a_{2 n+1}, \infty\right\}$.

(Here we have chosen a Cartan subspace $\mathfrak{a}$ of $\mathfrak{g}_{1}$ such that it consists of diagonal matrices.) This family gives us a monodromy representation $B_{N} \rightarrow S p\left(H^{1}\left(C_{a}, \mathbb{C}\right)\right)$. Note that, by $\mathrm{A}$ (see also $[\mathrm{KaS}]$ ) this monodromy representation has a Zariski dense image, in particular, the monodromy is infinite. From this we get a monodromy representation on the Jacobian of $C_{a}$ which we break into primitive parts:

$$
B_{N} \rightarrow H^{i}\left(\operatorname{Jac}\left(C_{a}\right), \mathbb{C}\right)_{\text {prim }} \simeq\left(\wedge^{i} H^{1}\left(C_{a}, \mathbb{C}\right)\right)_{\text {prim }} \quad i \in[1, n]
$$

Associated to this representation we obtain a local system $\mathcal{F}_{i}$ on $\mathfrak{g}_{1}^{r s}$. Note that the part $A[2]$ of $\pi_{1}^{K}\left(\mathfrak{g}_{1}^{r s}\right)$ acts trivially on $\mathcal{F}_{i}$.

Theorem 2.3. We have $\mathfrak{F}\left(\mathrm{IC}\left(\mathcal{O}_{2^{i} 1^{2 n+1-2 i}}, \mathcal{E}_{i}\right)\right)=\operatorname{IC}\left(\mathfrak{g}_{1}, \mathcal{F}_{i}\right), i=1, \ldots, n$.

\footnotetext{
${ }^{2}$ We have chosen this parametrization to get a clean statement for our theorem below.
} 


\section{Bijection of SETS}

In this section we show that

Proposition 3.1. There is a permutation s of the set $\{0, \ldots, n\}$ such that

$$
\mathfrak{F}\left(\mathrm{IC}\left(\mathcal{O}_{2^{k} 1^{2 n-2 k+1}}, \mathbb{C}\right)\right) \simeq \operatorname{IC}\left(\mathfrak{g}_{1}, \mathcal{L}_{s(k)}\right)
$$

for $k \in\{0, \ldots, n\}$.

Proposition 3.2. There is a permutation $s \in S_{n}$ such that

$$
\mathfrak{F}\left(\mathrm{IC}\left(\mathcal{O}_{2^{i} 1^{2 n-2 i+1}}, \mathcal{E}_{i}\right)\right) \simeq \mathrm{IC}\left(\mathfrak{g}_{1}, \mathcal{F}_{s(i)}\right), i \in[1, n] .
$$

Let us denote by $\operatorname{OGr}(s, 2 n+1)$ the variety of $s$-dimensional isotropic subspaces in $\mathbb{C}^{2 n+1}$ with respect to a non-degenerate bilinear form. We write $V_{i}$ for a vector subspace of $V=$ $\mathbb{C}^{2 n+1}$ of dimension $i$ and $V_{i}^{\perp}=\left\{x \in V \mid\left\langle x, V_{i}\right\rangle_{Q}=0\right\}$.

3.1. Proof of Proposition 3.1. Let $P$ be the parabolic subgroup in $G$ that stabilizes the partial flag $0 \subset V_{n}^{0} \subset V_{n}^{0 \perp} \subset V$, where $V_{n}^{0}=\operatorname{span}\left\{e_{1}, \ldots, e_{n}\right\}$ (here $\left(e_{i}\right)$ is a basis of $V$ such that $\left.\left\langle e_{i}, e_{j}\right\rangle_{Q}=\delta_{i+j, 2 n+2}\right)$. Let $P_{K}=K \cap P$ and

$$
E=\left\{x \in \mathfrak{g}_{1} \mid x V_{n}^{0 \perp}=0\right\} .
$$

By Reeder [R], we have the following resolution of singularities of $\overline{\mathcal{O}}_{2^{n}} 1$

$$
\pi_{2^{n} 1}: K \times{ }^{P_{K}} E \cong\left\{\left(x, 0 \subset V_{n} \subset V_{n}^{\perp} \subset V\right) \mid x \in \mathfrak{g}_{1}, x V_{n}^{\perp}=0\right\} \longrightarrow \overline{\mathcal{O}}_{2^{n} 1} .
$$

Lemma 3.3. The map $\pi_{2^{n} 1}$ is semi-small and we have

$$
\pi_{2^{n} 1 *} \mathbb{C}[-] \cong \bigoplus_{k=0}^{n} \mathrm{IC}\left(\mathcal{O}_{2^{k} 1^{2 n-2 k+1}}, \mathbb{C}\right) \text {. }
$$

Proof. Let $x \in \mathcal{O}_{2^{k} 1^{2 n-2 k+1}}$. Suppose that $V_{n} \in \pi_{2^{n} 1}^{-1}(x)$. Then $\operatorname{Im} x \subset V_{n}$ and $\operatorname{dim} \operatorname{Im} x=k$. We have that

$$
\begin{gathered}
\pi_{2^{n} 1}^{-1}(x) \cong\left\{n-k \text { dimensional isotropic subspaces of }(\operatorname{Im} x)^{\perp} / \operatorname{Im} x\right\} \\
\cong \operatorname{OGr}(n-k, 2 n-2 k+1) .
\end{gathered}
$$

Thus $\pi_{2^{n} 1}^{-1}(x)$ is irreducible and it is easy to check that $2 \operatorname{dim} \pi_{2^{n} 1}^{-1}(x)=(n-k)(n-k+1)=$ $\operatorname{codim}_{\overline{\mathcal{O}}_{2^{n}}} \mathcal{O}_{2^{k} 1^{2 n-2 k+1}}$. In the last equation we use the fact that $\operatorname{dim} \mathcal{O}_{2^{k} 1^{2 n-2 k+1}}=k(2 n+1-k)$. The lemma follows from the decomposition theorem.

Consider the map

$$
\check{\pi}_{2^{n} 1}: K \times{ }^{P_{K}} E^{\perp} \cong\left\{\left(x, 0 \subset V_{n} \subset V_{n}^{\perp} \subset V\right) \mid x \in \mathfrak{g}_{1}, x V_{n} \subset V_{n}^{\perp}\right\} \longrightarrow \mathfrak{g}_{1},
$$

where $E^{\perp}$ denotes the orthogonal complement of $E$ in $\mathfrak{g}_{1}$ with respect to the non-degenerate form on $\mathfrak{g}_{1}$ (given by the restriction of the Killing form on $\mathfrak{g}$ ). For $x \in \mathfrak{g}_{1}$, we have

$$
\check{\pi}_{2^{n} 1}^{-1}(x)=\left\{V_{n} \text { maximal isotropic subspace in } V \mid x V_{n} \subset V_{n}^{\perp}\right\} .
$$


For $x \in \mathfrak{g}_{1}^{r s}$ there are $2^{2 n}$ such maximal isotropic subspaces in $V$ and the centralizer $Z_{K}(x)$ acts simply-transitively on those subspaces (see [Re, $[\mathrm{BG}]$ ).

Consider the $K$-equivariant local system $\mathcal{L}=\left.\left(\check{\pi}_{2^{n} 1}\right)_{*} \mathbb{C}\right|_{\mathfrak{g}_{1}^{r s}}$ of rank $2^{2 n}$ on $\mathfrak{g}_{1}^{r s}$. Our first goal is to describe this local system. Fix $a \in \mathfrak{a}^{r s}$. The stalk $L:=\mathcal{L}_{a}$ carries an action of the $K$-equivariant fundamental group $\pi_{1}^{K}\left(\mathfrak{g}_{1}^{r s}, a\right)=Z_{K}(a) \rtimes B_{N}$. As $Z_{K}(a)$ acts simply transitively on $\check{\pi}_{2^{n} 1}^{-1}(x)$, as was remarked above, we can identify $L$ with $\mathbb{C}\left[Z_{K}(a)\right]=\mathbb{C}[A[2]]$. Furthermore, the action of $\pi_{1}^{K}\left(\mathfrak{g}_{1}^{r s}, a\right)$ factors through the Tits group $\widetilde{W}$ and it coincides with the canonical representation of $\widetilde{W}$ on $\mathbb{C}[A[2]]$. Let us recall the irreducible $K$-equivariant local systems $\mathcal{L}_{i}$ from $\$ 2$ and then

$$
\mathcal{L}=\bigoplus_{i=0}^{n} \mathcal{L}_{i}
$$

Lemma 3.4. We have

$$
\left(\check{\pi}_{2^{n} 1}\right)_{*} \mathbb{C}[-]=\operatorname{IC}\left(\mathfrak{g}_{1}, \mathcal{L}\right)=\bigoplus_{i=0}^{n} \operatorname{IC}\left(\mathfrak{g}_{1}, \mathcal{L}_{i}\right) .
$$

In particular, the map $\check{\pi}_{2^{n} 1}$ is small.

Proof. By the decomposition theorem, $\bigoplus_{i=0}^{n} \mathrm{IC}\left(\mathfrak{g}_{1}, \mathcal{L}_{i}\right)$ is a direct summand of $\left(\check{\pi}_{2^{n}}\right)_{*} \mathbb{C}[-]$. On the other hand, since, by the functoriality of the Fourier transform,

$$
\mathfrak{F}\left(\left(\pi_{2^{n} 1}\right)_{*} \mathbb{C}[-]\right) \simeq\left(\check{\pi}_{2^{n} 1}\right)_{*} \mathbb{C}[-],
$$

the decomposition in (3.2) implies that $\left(\check{\pi}_{2^{n} 1}\right)_{*} \mathbb{C}[-]$ has exactly $n+1$ irreducible summands. The lemma follows from (3.4).

Proposition 3.1 follows from (3.2), (3.5) and (3.6).

3.2. Proof of Proposition 3.2. Let $V=\mathbb{C}^{2 n+1}$ and $W=V \oplus \mathbb{C}$. For any $x \in \mathfrak{g}_{1}$, consider the following two quadrics in $\mathbb{P}(W)$ :

$$
\widetilde{Q}(v, a)=\langle v, v\rangle_{Q}=0 \text { and } \widetilde{Q}_{x}(v, a)=\langle v, x v\rangle_{Q}+a^{2}=0 .
$$

Define

$$
F=\left\{\left(x, W_{n} \subset W\right) \mid x \in \mathfrak{g}_{1}, \operatorname{dim} W_{n}=n, \quad \mathbb{P}\left(W_{n}\right) \subset \widetilde{Q} \cap \widetilde{Q}_{x}\right\} .
$$

The map $W \rightarrow W$ given by $(v, a) \mapsto(v,-a)$ induces an involution $\sigma$ on $F$ and the set $F^{\sigma}$ of fixed points is equal to

$$
F^{\sigma}=\left\{\left(x, V_{n} \subset V\right) \mid x \in \mathfrak{g}_{1}, V_{n} \text { maximal isotropic, } x V_{n} \subset V_{n}^{\perp}\right\} \cong K \times{ }^{P_{K}} E^{\perp}(\text { see (3.3) }) .
$$

Note that for $x \in \mathfrak{g}_{1}^{r s}$, the pencil of quadrics spanned by $\widetilde{Q}$ and $\widetilde{Q}_{x}$ is non-degenerate and contains exactly $2 n+2$ singular elements, namely, the quadric $\widetilde{Q}$ at infinity and the $2 n+1$ quadrics $\lambda_{i} \widetilde{Q}-\widetilde{Q}_{x}$, where $\lambda_{1}, \ldots, \lambda_{2 n+1}$ are the roots of $p(t)=\operatorname{det}(t \cdot \mathrm{id}-x)$.

We denote by $\pi: F \rightarrow \mathfrak{g}_{1}$ the natural projection. The fiber $F_{x}=\pi^{-1}(x)$ of $\pi$ over $x \in \mathfrak{g}_{1}^{r s}$ is the Fano variety of $(n-1)$-dimensional subspaces contained in the smooth complete 
intersection $\widetilde{Q} \cap \widetilde{Q}_{x}$. According to $[\mathrm{Re}], F_{x}$ is a torsor over $\operatorname{Jac}\left(C_{x}\right)$, where $C_{x}$ is the smooth projective hyperelliptic curve with affine equation:

$$
y^{2}=\prod_{i=1}^{2 n+1}\left(t-\lambda_{i}\right) .
$$

Moreover, the action of the involution $\sigma$ on $F_{x}$ is compatible with the inversion on $\operatorname{Jac}\left(C_{x}\right)$. In particular, the set $F_{x}^{\sigma}$ of fixed points is a $\operatorname{Jac}\left(C_{x}\right)$ [2]-torsor, where $\operatorname{Jac}\left(C_{x}\right)[2]$ consists of 2-torsion points of $\operatorname{Jac}\left(C_{x}\right)$.

The discussion above has the following relative version. Namely, let $\pi^{C}: C \rightarrow \mathfrak{g}_{1}^{r s}$ be the family of curves $C_{x}$ and let $\operatorname{Jac}(C) \rightarrow \mathfrak{g}_{1}^{r s}$ denote the corresponding relative Jacobian. Let $\left.F\right|_{\mathfrak{g}_{1}^{r s}} \rightarrow \mathfrak{g}_{1}^{r s}$ be the family of Fano varieties of $(n-1)$-dimensional subspaces contained in the smooth complete intersection $\tilde{Q} \cap \tilde{Q}_{x}$. Then $\operatorname{Jac}(C)$ acts naturally on $\left.F\right|_{\mathfrak{g}_{1}^{r s}}$, and $\left.F\right|_{\mathfrak{g}_{1}^{r s}}$ is a $\operatorname{Jac}(C)$-torsor under this action. Similarly, $\operatorname{Jac}(C)[2]$ acts on $F^{\sigma}$, and $F^{\sigma}$ is a $\operatorname{Jac}(C)[2]$-torsor under this action. We have the following observation.

Lemma 3.5. (1) The action map $\operatorname{Jac}(C) \times\left.\left._{\mathfrak{g}_{1}^{r s}} F^{\sigma}\right|_{\mathfrak{g}_{1}^{r s}} \rightarrow F\right|_{\mathfrak{g}_{1}^{r s}}$ factors through an isomorphism

$$
\left(\operatorname{Jac}(C) \times\left._{\mathfrak{g}_{1}^{r s}} F^{\sigma}\right|_{\mathfrak{g}_{1}^{r s}}\right) /\left.\operatorname{Jac}(C)[2] \simeq F\right|_{\mathfrak{g}_{1}^{r s}}
$$

Here $\operatorname{Jac}(C)[2]$ acts on $\operatorname{Jac}(C) \times\left._{\mathfrak{g}_{1}^{r s}} F^{\sigma}\right|_{\mathfrak{g}_{1}^{r s}}$ via the diagonal action.

(2) For any $a \in \mathfrak{g}_{1}^{r s}$, there is a canonical isomorphism

$$
H^{i}\left(\operatorname{Jac}\left(C_{a}\right), \mathbb{C}\right) \simeq H^{i}\left(\left(\operatorname{Jac}\left(C_{a}\right) \times F_{a}^{\sigma}\right) / \operatorname{Jac}\left(C_{a}\right)[2], \mathbb{C}\right)
$$

compatible with the monodromy actions of $\pi_{1}^{K}\left(\mathfrak{g}_{1}^{r s}, a\right)$ on both sides.

Proof. (1) is clear. For (2), we observe that, by Künneth formula, we have

$$
H^{i}\left(\left(\operatorname{Jac}\left(C_{a}\right) \times F_{a}^{\sigma}\right) / \operatorname{Jac}\left(C_{a}\right)[2], \mathbb{C}\right) \simeq\left(H^{i}\left(\operatorname{Jac}\left(C_{a}\right), \mathbb{C}\right) \otimes H^{0}\left(F_{a}^{\sigma}, \mathbb{C}\right)\right)^{\operatorname{Jac}\left(C_{a}\right)[2]}
$$

where the right hand side is the $\left(\operatorname{Jac}\left(C_{a}\right)[2]\right)$-fixed vectors in $H^{i}\left(\operatorname{Jac}\left(C_{a}\right), \mathbb{C}\right) \otimes H^{0}\left(F_{a}^{\sigma}, \mathbb{C}\right)$. Since the action of $\operatorname{Jac}\left(C_{a}\right)[2]$ on $H^{i}\left(\operatorname{Jac}\left(C_{a}\right), \mathbb{C}\right)$ is trivial $]^{3}$ we have

$$
\left(H^{i}\left(\operatorname{Jac}\left(C_{a}\right), \mathbb{C}\right) \otimes H^{0}\left(F_{a}^{\sigma}, \mathbb{C}\right)\right)^{\operatorname{Jac}\left(C_{a}\right)[2]} \simeq H^{i}\left(\operatorname{Jac}\left(C_{a}\right), \mathbb{C}\right),
$$

Combining (3.8) and (3.9), we obtain a canonical isomorphism

$$
H^{i}\left(\left(\operatorname{Jac}\left(C_{a}\right) \times F_{a}^{\sigma}\right) / \operatorname{Jac}\left(C_{a}\right)[2], \mathbb{C}\right) \simeq H^{i}\left(\operatorname{Jac}\left(C_{a}\right), \mathbb{C}\right) .
$$

Since the isomorphisms in (3.8), (3.9) are compatible with the monodromy actions, so is the composition in (3.10). Thus (2) follows.

It follows immediately that

Corollary 3.6. There is a canonical isomorphism $H^{i}\left(F_{a}, \mathbb{C}\right) \simeq H^{i}\left(\operatorname{Jac}\left(C_{a}\right), \mathbb{C}\right)$ compatible with the monodromy actions of $\pi_{1}^{K}\left(\mathfrak{g}_{1}^{r s}, a\right)$.

\footnotetext{
${ }^{3}$ To see this, we observe that the action of $\operatorname{Jac}\left(C_{a}\right)[2]$ on $H^{i}\left(\operatorname{Jac}\left(C_{a}\right)\right)$ is the restriction of the action of $\operatorname{Jac}\left(C_{a}\right)$ on $H^{i}\left(\operatorname{Jac}\left(C_{a}\right)\right)$. Since $\operatorname{Jac}\left(C_{a}\right)$ is connected the latter action is trivial.
} 
We use the corollary above to study the monodormy of the family of Fano varieties $\left.F\right|_{\mathfrak{g}_{1}^{r s}} \rightarrow$ $\mathfrak{g}_{1}^{r s}$. To begin with, we observe that over the Kostant section $\kappa: \mathfrak{c}^{r s} \hookrightarrow \mathfrak{g}_{1}^{r s}$, the family $\pi^{C}: C \rightarrow \mathfrak{g}_{1}^{r s}$ is the universal family of hyperelliptic curves of genus $n$. As mentioned before, the monodromy representation of $\pi_{1}^{K}\left(\mathfrak{g}_{1}^{r s}, a\right)$ on $H^{1}\left(C_{a}, \mathbb{C}\right)$ is irreducible and the image of $\pi_{1}^{K}\left(\mathfrak{g}_{1}^{r s}, a\right) \rightarrow S p\left(H^{1}\left(C_{a}, \mathbb{C}\right)\right)$ is Zariski dense. This fact together with the corollary above implies that the monodromy representation of $\pi_{1}^{K}\left(\mathfrak{g}_{1}^{r s}, a\right)$ on

$$
H^{i}\left(F_{a}, \mathbb{C}\right)_{\text {prim }} \simeq H^{i}\left(\operatorname{Jac}\left(C_{a}\right), \mathbb{C}\right)_{\text {prim }} \simeq\left(\wedge^{i} H^{1}\left(C_{a}, \mathbb{C}\right)\right)_{\text {prim }}
$$

is irreducible for $i=1, \ldots, n$, moreover, the corresponding monodromy group is infinite. Thus the corresponding irreducible $K$-equivariant local systems on $\mathfrak{g}_{1}^{r s}$ are

$$
\left(\mathrm{R}^{i} \pi_{*} \mathbb{C}_{\mathfrak{g}_{1}^{r s}}\right)_{\text {prim }}=\mathcal{F}_{i}
$$

where $\mathcal{F}_{i}$ is the local system defined in $\$ 2.3$. We have

$$
\operatorname{dim} \mathcal{F}_{i}=\left(\begin{array}{c}
2 n \\
i
\end{array}\right)-\left(\begin{array}{c}
2 n \\
i-2
\end{array}\right)
$$

and $\mathcal{F}_{i} \nsucceq \mathcal{F}_{j}$ for $i \neq j$.

Recall the local systems $\left\{\mathcal{L}_{i}\right\}_{i=0, \ldots, n}$ defined in $\$ 2.3$. Since each $\mathcal{L}_{i}$ has finite monodromy, we have $\mathcal{F}_{i} \not \mathcal{L}_{j}$ for all $i, j$. As there is only one nontrivial irreducible $K$-equivariant local system $\mathcal{E}_{i}$ on each $\mathcal{O}_{2^{i} 1^{2 n-2 i+1}}(i \geq 1)$, Proposition 3.2 follows from Proposition 3.1 and the following proposition

Proposition 3.7. For $i=1, \ldots, n, \mathfrak{F}\left(\mathrm{IC}\left(\mathfrak{g}_{1}, \mathcal{F}_{i}\right)\right)$ is supported on $\overline{\mathcal{O}}_{2^{n} 1}$.

Proof. To begin with, we show that the variety $F$ defined in (3.7) is isomorphic to

$$
\begin{gathered}
\left\{\left(x, V_{n}, \alpha\right) \mid x \in \mathfrak{g}_{1}, V_{n} \text { maximal isotropic in } V, \alpha \in V_{n}^{*},\right. \\
\left.\left\langle v, x v^{\prime}\right\rangle+\alpha(v) \alpha\left(v^{\prime}\right)=0 \text { for all } v, v^{\prime} \in V_{n}\right\} .
\end{gathered}
$$

Consider the projection maps $p_{1}: W=V \oplus \mathbb{C} \rightarrow V$ and $p_{2}: W=V \oplus \mathbb{C} \rightarrow \mathbb{C}$. Let $\left(x, W_{n}\right) \in F$ and let $V_{n}=p_{1}\left(W_{n}\right)$. It is easy to see that $\left.p_{1}\right|_{W_{n}}: W_{n} \rightarrow V_{n}$ is an isomorphism. Moreover $V_{n} \subset V_{n}^{\perp} \subset V$. Let $\alpha: V_{n} \rightarrow \mathbb{C}$ be defined by $\alpha(v)=p_{2} \circ\left(\left.p_{1}\right|_{W_{n}}\right)^{-1} v$. One checks readily that $\left(x, V_{n}, \alpha\right)$ belongs to the set in (3.12). Conversely, given $\left(x, V_{n}, \alpha\right)$, we let $W_{n}=\left\{(v, \alpha(v)) \mid v \in V_{n}\right\}$. Then $\left(x, W_{n}\right) \in F$. It follows that $F$ is smooth.

Since $\pi: F \rightarrow \mathfrak{g}_{1}$ is proper, the decomposition theorem and (3.11) imply that $\operatorname{IC}\left(\mathfrak{g}_{1}, \mathcal{F}_{i}\right)$ (up to shift) is a summand of $\pi_{*} \mathbb{C}_{F}, i=1, \ldots, n$. Thus it is enough to show that $\mathfrak{F}\left(\pi_{*} \mathbb{C}_{F}\right)$ is supported on $\overline{\mathcal{O}}_{2^{n} 1}$.

Recall the maps $\pi_{2^{n} 1}: K \times^{P_{K}} E \rightarrow \overline{\mathcal{O}}_{2^{n} 1}$ and $\check{\pi}_{2^{n} 1}: K \times^{P_{K}} E^{\perp}$ defined in (3.1) and (3.3). Let $q: K / P_{K} \times \mathfrak{g}_{1} \simeq K \times{ }^{P_{K}} \mathfrak{g}_{1} \rightarrow K \times{ }^{P_{K}}\left(\mathfrak{g}_{1} / E^{\perp}\right)$ denote the quotient map. Note that the non-degenerate invariant form on $\mathfrak{g}_{1}$ induces isomorphisms $E \simeq\left(\mathfrak{g}_{1} / E^{\perp}\right)^{*}, \mathfrak{g}_{1} \simeq \mathfrak{g}_{1}^{*}$, and under these isomorphisms, the dual map of $q$ can be identified with the natural embedding

$$
\check{q}: K \times{ }^{P_{K}} E \rightarrow K \times{ }^{P_{K}} \mathfrak{g}_{1} \text {. }
$$

Let

$$
\begin{gathered}
\bar{F}=\left\{\left(\bar{x}, V_{n}, \alpha\right) \mid \bar{x} \in \mathfrak{g}_{1} /\left\{x \in \mathfrak{g}_{1} \mid x V_{n} \subset V_{n}^{\perp}\right\}, V_{n} \text { maximal isotropic in } V, \alpha \in V_{n}^{*},\right. \\
\left.\left\langle v, x v^{\prime}\right\rangle+\alpha(v) \alpha\left(v^{\prime}\right)=0 \text { for all } v, v^{\prime} \in V_{n} \text { and } x \in \mathfrak{g}_{1} \text { any lift of } \bar{x}\right\} .
\end{gathered}
$$


We have the following Cartesian diagram:

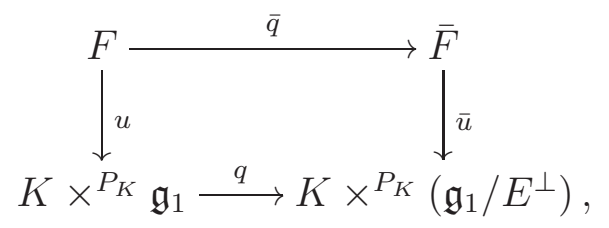

where $u:\left(x, V_{n}, \alpha\right) \mapsto\left(V_{n}, x\right), \bar{q}:\left(x, V_{n}, \alpha\right) \mapsto\left(\bar{x}, V_{n}, \alpha\right)$ and $\bar{u}:\left(\bar{x}, V_{n}, \alpha\right) \mapsto\left(V_{n}, \bar{x}\right)$.

Let us write $p r: K \times^{P_{K}} \mathfrak{g}_{1} \rightarrow \mathfrak{g}_{1}$ for the natural projection. We have that, by functoriality of the Fourier transform,

$$
\begin{aligned}
\mathfrak{F}\left(\pi_{*} \mathbb{C}_{F}\right)= & \mathfrak{F}\left(p r_{*} u_{*} \mathbb{C}_{F}\right)=\mathfrak{F}\left(p r_{*} u_{*} \bar{q}^{*} \mathbb{C}_{\bar{F}}\right)=\mathfrak{F}\left(p r_{*} q^{*} \bar{u}_{*} \mathbb{C}_{\bar{F}}\right) \\
& =p r_{*} \check{q}_{*} \mathfrak{F}\left(\bar{u}_{*} \mathbb{C}_{\bar{F}}\right)=\pi_{2^{n} 1 *} \mathfrak{F}\left(\bar{u}_{*} \mathbb{C}_{\bar{F}}\right) .
\end{aligned}
$$

It follows that $\mathfrak{F}\left(\pi_{*} \mathbb{C}_{F}\right)$ is supported on $\overline{\mathcal{O}}_{2^{n} 1}$.

\section{Proof of the Main theorems}

In this section we prove Theorem 2.2 below, but give a proof of Theorem 2.3 only for orbits $\mathcal{O}_{2^{i} 1^{2 n+1-2 i}}$ when $i$ is even. We defer the case of odd $i$ to [CVX1, Proposition 5.4].

4.1. Proof of Theorem 2.3 for $i$ even. Consider the maps

$$
\begin{gathered}
v:\left\{\left(x, 0 \subset V_{n-1} \subset V_{n} \subset V_{n}^{\perp} \subset V_{n-1}^{\perp} \subset V=\mathbb{C}^{2 n+1}\right) \mid x \in \mathfrak{g}_{1}, x V_{n}^{\perp}=0, x V_{n-1}^{\perp} \subset V_{n-1}\right\}:=Y \\
\qquad \mathcal{N}_{1}, \\
\check{v}:\left\{\left(x, 0 \subset V_{n-1} \subset V_{n} \subset V_{n}^{\perp} \subset V_{n-1}^{\perp} \subset V=\mathbb{C}^{2 n+1}\right) \mid x \in \mathfrak{g}_{1}, x V_{n-1} \subset V_{n}^{\perp}\right\} \rightarrow \mathfrak{g}_{1} .
\end{gathered}
$$

We have that

$$
\operatorname{dim} Y=n^{2}+2 n-2, \quad \operatorname{Im} v=\overline{\mathcal{O}}_{2^{n} 1}
$$

and

$$
\mathfrak{F}\left(v_{*} \mathbb{C}[-]\right)=\check{v}_{*} \mathbb{C}[-]
$$

In the following we prove the theorem by studying the decompositions of $v_{*} \mathbb{C}[-]$ and $\check{v}_{*} \mathbb{C}[-]$.

We first study the decomposition of $\check{v}_{*} \mathbb{C}[-]$. Since in the decomposition of $v_{*} \mathbb{C}[-]$ only IC complexes supported on $\mathcal{O}_{2^{i} 1^{2 n+1-2 i}}$ appear and the Fourier transform of such complexes all have full support (see Proposition 3.1 and Proposition 3.2), we conclude that all IC complexes appearing in the decomposition of $\check{v}_{*} \mathbb{C}[-]$ are supported on all of $\mathfrak{g}_{1}$.

Let $F$ be the smooth variety introduced in (3.7). Recall its equivalent definition in (3.12) and the involution $\sigma$ on $F$. Let

$$
Z=\left\{\left(x, 0 \subset V_{n} \subset V_{n}^{\perp} \subset V\right) \mid x \in \mathfrak{g}_{1}, \operatorname{rank}\left(\bar{x}: V_{n} \rightarrow V / V_{n}^{\perp}\right) \leq 1\right\}
$$

and

$$
Z_{0}=\left\{\left(x, 0 \subset V_{n} \subset V_{n}^{\perp} \subset V\right) \mid x \in \mathfrak{g}_{1}, \operatorname{rank}\left(\bar{x}: V_{n} \rightarrow V / V_{n}^{\perp}\right)=0\right\} .
$$

We have $\operatorname{dim} Z=\operatorname{dim} \mathfrak{g}_{1}+n$ and $\operatorname{dim} Z_{0}=\operatorname{dim} \mathfrak{g}_{1}$. Consider the map

$$
f: F \rightarrow Z,\left(x, V_{n}, \alpha\right) \mapsto\left(x, V_{n}\right) .
$$


Then $f$ is a branched double cover with branch locus $Z_{0}$ 4. Let $Z^{0}:=Z-Z_{0}$. Then $f^{0}:=\left.f\right|_{Z^{0}}: F \times{ }_{Z} Z^{0} \rightarrow Z^{0}$ is a double cover and we have $f_{*}^{0} \mathbb{C}=\mathbb{C} \oplus \mathcal{E}$, where $\mathcal{E}=\left(f_{*}^{0} \mathbb{C}\right)^{\sigma=-i d}$ is a rank one local system on $Z^{0}$. It follows that

$$
f_{*} \mathbb{C}_{F}[\operatorname{dim} F]=\operatorname{IC}(Z, \mathbb{C}) \oplus \operatorname{IC}(Z, \mathcal{E}) .
$$

Let $a \in \mathfrak{a}^{r s}$ and let $p r: Z \rightarrow \mathfrak{g}_{1}$ be the natural projection. By (4.2), we have

$$
\left.p r_{*} \mathrm{IC}(Z, \mathbb{C})[-\operatorname{dim} F]\right|_{a} \simeq H^{*}\left(F_{a}, \mathbb{C}\right)^{\sigma=i d},\left.\quad p r_{*} \operatorname{IC}(Z, \mathcal{E})[-\operatorname{dim} F]\right|_{a} \simeq H^{*}\left(F_{a}, \mathbb{C}\right)^{\sigma=-i d} .
$$

Choosing an isomorphism $\operatorname{Jac}\left(C_{a}\right) \simeq F_{a}$, we may identify $\sigma$ with the inversion on $\operatorname{Jac}\left(C_{a}\right)$. Therefore

$$
H^{*}\left(F_{a}, \mathbb{C}\right)^{\sigma=i d} \simeq \oplus_{i=2 j} \wedge^{i} H^{1}\left(C_{a}, \mathbb{C}\right)[-i], H^{*}\left(F_{a}, \mathbb{C}\right)^{\sigma=-i d} \simeq \oplus_{i=2 j+1} \wedge^{i} H^{1}\left(C_{a}, \mathbb{C}\right)[-i] .
$$

This implies that

$$
\begin{gathered}
\mathrm{IC}\left(\mathfrak{g}_{1}, \oplus_{i=2 j} \oplus_{k=0}^{j} \mathcal{F}_{2 k}[-i]\right) \text { (resp. } \mathrm{IC}\left(\mathfrak{g}_{1}, \oplus_{i=2 j+1} \oplus_{k=0}^{j} \mathcal{F}_{2 k+1}[-i]\right) \text { appears } \\
\text { in } p r_{*} \mathrm{IC}(Z, \mathbb{C}) \text { (resp. } p r_{*} \mathrm{IC}(Z, \mathcal{E}) \text { ) as a direct summand (up to shift). }
\end{gathered}
$$

Moreover, these are the only IC complexes with full support appearing in the decomposition.

We have the following factorization of $\check{v}$

$$
\left\{\left(x, 0 \subset V_{n-1} \subset V_{n} \subset V_{n}^{\perp} \subset V_{n-1}^{\perp} \subset V=\mathbb{C}^{2 n+1}\right) \mid x V_{n-1} \subset V_{n}^{\perp}\right\} \stackrel{\check{v}_{1}}{\longrightarrow} Z \stackrel{\check{v}_{2}}{\longrightarrow} \mathfrak{g}_{1},
$$

where $\check{v}_{1}:\left(x, V_{n-1} \subset V_{n}\right) \mapsto\left(x, V_{n}\right)$ and $\check{v}_{2}:\left(x, V_{n}\right) \mapsto x$. Note that $\left.\check{v}_{2}\right|_{Z_{0}}: Z_{0} \rightarrow \mathfrak{g}_{1}$ is equal to the map $\check{\pi}_{2^{n} 1}$ in $(3.3)$.

The map $\check{v}_{1}$ is one-to-one over $Z-Z / \sqrt{5}$ and is a $\mathbb{P}^{n-1}$-bundle over $Z_{0}$. It follows that

$$
\check{v}_{1 *} \mathbb{C}[-]=\operatorname{IC}(Z, \mathbb{C}) \oplus \bigoplus_{a=0}^{n-2} \mathbb{C}_{Z_{0}}[-][n-2-2 a]
$$

Since all IC complexes appearing in the decomposition of $\check{v}_{*} \mathbb{C}[-]$ are supported on all of $\mathfrak{g}_{1}$, Lemma 3.4 and (4.3) imply that

$$
\check{v}_{*} \mathbb{C}[-] \cong \check{v}_{2 *} \check{v}_{1 *} \mathbb{C}[-] \cong \bigoplus_{k=0}^{\left[\frac{n}{2}\right]} \operatorname{IC}\left(\mathfrak{g}_{1}, \bigoplus_{s=0}^{k} \mathcal{F}_{2 s}\right)[ \pm(n-2 k)] \oplus \bigoplus_{a=0}^{n-2} \operatorname{IC}\left(\mathfrak{g}_{1}, \bigoplus_{s=0}^{n} \mathcal{L}_{s}\right)[n-2-2 a]
$$

here $\mathcal{F}_{0}=\mathbb{C}$.

We now study the decomposition of $v_{*} \mathbb{C}[-]$. Our goal is to prove the following

$$
\begin{gathered}
v_{*} \mathbb{C}\left[n^{2}+2 n-2\right] \cong \bigoplus_{a=0}^{n-2} \bigoplus_{i=0}^{n} \operatorname{IC}\left(\mathcal{O}_{2^{i} 1^{2 n-2 i+1}}, \mathbb{C}\right)[n-2-2 a] \\
\oplus \bigoplus_{i=1}^{\left[\frac{n}{2}\right]} \bigoplus_{a_{i}=0}^{n-2 i} \operatorname{IC}\left(\mathcal{O}_{2^{2 i} 1^{2 n+1-4 i}}, \mathcal{E}_{2 i}\right)\left[n-2 i-2 a_{i}\right] \oplus \bigoplus_{a=0}^{n} \operatorname{IC}\left(\mathcal{O}_{1^{2 n+1}}, \mathbb{C}\right)[n-2 a] .
\end{gathered}
$$

\footnotetext{
${ }^{4}$ In fact, the map $f: F \rightarrow Z$ realizes $Z$ as the (GIT) quotient $F / \sigma$.

${ }^{5}$ The inverse is given by $\left(x, V_{n}\right) \rightarrow\left(x, V_{n-1} \subset V_{n}\right)$ where $V_{n-1}:=\operatorname{Ker}\left(\bar{x}: V_{n} \rightarrow V / V_{n}^{\perp}\right)$.
} 
Taking Fourier transform of (4.4) and using Proposition 3.1, we see that

$$
\mathfrak{F}\left(\bigoplus_{a=0}^{n-2} \mathrm{IC}\left(\mathfrak{g}_{1}, \bigoplus_{s=0}^{n} \mathcal{L}_{s}\right)[n-2-2 a]\right) \simeq \bigoplus_{a=0}^{n-2} \bigoplus_{i=0}^{n} \mathrm{IC}\left(\mathcal{O}_{2^{i} 1^{2 n-2 i+1}}, \mathbb{C}\right)[n-2-2 a]
$$

is a direct summand of $v_{*} \mathbb{C}[-]$.

Moreover, these contain all the $\operatorname{IC}\left(\mathcal{O}_{2^{i} 1^{2 n-2 i+1}}, \mathbb{C}\right), i \geq 1$, that appears in the decomposition of $v_{*} \mathbb{C}[-]$. Now we determine those $\operatorname{IC}\left(\mathcal{O}_{2^{i} 1^{2 n-2 i+1}}, \mathcal{E}_{i}\right), i \geq 1$, that appears in the decomposition of $v_{*} \mathbb{C}[-]$.

Let $x_{i} \in \mathcal{O}_{2^{i} 1^{2 n-2 i+1}}, i \in[1, n]$. We have that $v^{-1}\left(x_{i}\right)$ is a quadric bundle over $v_{0}^{-1}\left(x_{i}\right) \cong$ $\operatorname{OGr}(n-i, 2 n-2 i+1)$, with fibers quadric of the form $\sum_{k=1}^{i} a_{k}^{2}=0$ in $\mathbb{P}^{n-1}=\left\{\left[a_{1}: \ldots: a_{n}\right]\right\}$, and

$$
2 \operatorname{dim} v^{-1}\left(x_{i}\right)=\operatorname{codim}_{E} \mathcal{O}_{x_{i}}+n-2=2(n-2)+(n-i)(n-i+1) .
$$

Here $v_{0}=\pi_{2^{n} 1}$ is the map defined in (3.1). We have that (see (3.2))

$$
v_{0 *} \mathbb{C}\left[n^{2}+n\right] \cong \bigoplus_{i=0}^{n} \mathrm{IC}\left(\mathcal{O}_{2^{i} 1^{2 n-2 i+1}}, \mathbb{C}\right)
$$

which implies that

$$
H^{k}\left(v_{0}^{-1}\left(x_{j}\right), \mathbb{C}\right) \cong \mathcal{H}_{x_{j}}^{k-n^{2}-n}\left(\bigoplus_{i=0}^{n} \mathrm{IC}\left(\mathcal{O}_{2^{i} 1^{2 n-2 i+1}}, \mathbb{C}\right)\right)
$$

We have $H^{\text {odd }}\left(v_{0}^{-1}\left(x_{j}\right), \mathbb{C}\right)=H^{\text {odd }}\left(v^{-1}\left(x_{j}\right), \mathbb{C}\right)=0$ and

$$
H^{2 k}\left(v^{-1}\left(x_{j}\right), \mathbb{C}\right) \cong \bigoplus_{a=0}^{n-2} H^{2 a}\left(Q_{j}, \mathbb{C}\right) \otimes H^{2 k-2 a}\left(v_{0}^{-1}\left(x_{j}\right), \mathbb{C}\right),
$$

where $Q_{j}$ is a quadric of the form $\sum_{s=1}^{j} a_{s}^{2}=0$ in $\mathbb{P}^{n-1}=\left\{\left[a_{1}: \ldots: a_{n}\right]\right\}$. Note that $H^{2 a}\left(Q_{j}, \mathbb{C}\right)=\mathbb{C}$ for $0 \leq a \leq n-2$ if $j$ is odd, or if $j$ is even and $2 a \neq 2 n-j-2$, and for $j$ even, $H^{2 n-j-2}\left(Q_{j}, \mathbb{C}\right) \cong \mathbb{C} \oplus H_{\text {prim }}^{2 n-j-2}\left(Q_{j}, \mathbb{C}\right)$, where $\operatorname{dim} H_{\text {prim }}^{2 n-j-2}\left(Q_{j}, \mathbb{C}\right)=1$. Thus

$$
\begin{aligned}
& H^{2 k}\left(v^{-1}\left(x_{j}\right), \mathbb{C}\right) \cong \bigoplus_{a=0}^{n-2} H^{2 k-2 a}\left(v_{0}^{-1}\left(x_{j}\right), \mathbb{C}\right) \text { if } j \text { is odd } \\
& H^{2 k}\left(v^{-1}\left(x_{j}\right), \mathbb{C}\right) \cong \bigoplus_{a=0}^{n-2} H^{2 k-2 a}\left(v_{0}^{-1}\left(x_{j}\right), \mathbb{C}\right) \oplus H^{2 k-2 n+j+2}\left(v_{0}^{-1}\left(x_{j}\right), \mathbb{C}\right) \text { if } j \text { is even. }
\end{aligned}
$$

It follows from (4.7) that

$$
\mathcal{H}_{x_{j}}^{2 k-n^{2}-2 n+2} \bigoplus_{a=0}^{n-2} \bigoplus_{i=0}^{n} \mathrm{IC}\left(\mathcal{O}_{2^{i} 1^{2 n-2 i+1}}, \mathbb{C}\right)[n-2-2 a] \cong \bigoplus_{a=0}^{n-2} H^{2 k-2 a}\left(v_{0}^{-1}\left(x_{j}\right), \mathbb{C}\right)
$$

Thus (4.10) together with (4.8) implies that if $j$ is odd, then

$$
\mathcal{H}_{x_{j}}^{2 k} \bigoplus_{a=0}^{n-2} \bigoplus_{i=0}^{n} \mathrm{IC}\left(\mathcal{O}_{2^{i} 1^{2 n-2 i+1}}, \mathbb{C}\right)[n-2-2 a] \cong \mathcal{H}_{x_{j}}^{2 k}\left(v_{*} \mathbb{C}[-]\right)
$$


In view of (4.6), we conclude that $\mathrm{IC}\left(\mathcal{O}_{2^{j} 1^{2 n+1-2 j}}, \mathcal{E}_{j}\right), j$ odd, does not appear in the decomposition of $v_{*} \mathbb{C}[-]$.

By the discussion above, we can assume that

$$
\begin{gathered}
v_{*} \mathbb{C}[-]=\bigoplus_{a=0}^{n-2} \bigoplus_{i=0}^{n} \operatorname{IC}\left(\mathcal{O}_{2^{i} 1^{2 n-2 i+1}}, \mathbb{C}\right)[n-2-2 a] \\
\oplus \bigoplus_{i=1}^{\left[\frac{n}{2}\right]} \bigoplus_{a_{i}=0}^{k_{i}} \operatorname{IC}\left(\mathcal{O}_{2^{2 i} 1^{2 n+1-4 i}}, \mathcal{E}_{2 i}^{\oplus m_{a_{i}}^{i}}\right)\left[k_{i}-2 a_{i}\right] \oplus \cdots
\end{gathered}
$$

where the $m_{a_{i}}^{i}$ 's are to be determined and $\cdots$ is a sum of $\mathbb{C}_{\{0\}}[-]$. We show that

$$
k_{i}=n-2 i, m_{0}^{i}=m_{n-2 i}^{i}=1 .
$$

Note that in (4.9),$H^{2 k-2 n+j+2}\left(v_{0}^{-1}\left(x_{j}\right), \mathbb{C}\right) \neq 0$ if and only if $0 \leq 2 k-2 n+j+2 \leq$ $(n-j)(n-j+1)$, i.e. if and only if

$$
2 n-j-2 \leq 2 k \leq(n-j)(n-j+1)+2 n-j-2,
$$

since $\operatorname{dim} v_{0}^{-1}\left(x_{j}\right)=(n-j)(n-j-1) / 2$. Thus for all $l>(n-2 i)(n-2 i+1)+2 n-2 i-2$, we must have

$$
\mathcal{H}_{x_{2 i}}^{l-n^{2}-2 n+2} \bigoplus_{a_{i}=0}^{k_{i}} \mathrm{IC}\left(\mathcal{O}_{2^{2 i} 1^{2 n+1-4 i}}, \mathcal{E}_{2 i}^{\oplus m_{a_{i}}^{i}}\right)\left[k_{i}-2 a_{i}\right]=0,
$$

i.e. for all $l>n-2 i-\operatorname{dim} \mathcal{O}_{2^{2 i} 1^{2 n+1-4 i}}, \mathcal{H}_{x_{2 i}}^{l} \bigoplus_{a_{i}=0}^{k_{i}} \mathrm{IC}\left(\mathcal{O}_{2^{2 i} 1^{2 n+1-4 i}}, \mathcal{E}_{2 i}^{\oplus m_{a_{i}}^{i}}\right)\left[k_{i}-2 a_{i}\right]=0$. Thus $k_{i} \leq n-2 i$. It remains to show that

$$
m_{0}^{i}=m_{n-2 i}^{i}=1 .
$$

We argue using induction on $1 \leq i \leq\left[\frac{n}{2}\right]$. Consider $i=\left[\frac{n}{2}\right]$. If $n$ is even, then it is easy to see that $\operatorname{IC}\left(\mathcal{O}_{2^{n}}, \mathcal{E}_{n}\right)$ is a direct summand. Assume that $n$ is odd. Take $j=n-1$ in (4.9) we get $H^{2 k-2 n+j+2}\left(v_{0}^{-1}\left(x_{j}\right), \mathbb{C}\right) \neq 0$ if and only if $2 k=n+1, n-1$ (we have $v_{0}^{-1}\left(x_{n-1}\right)$ is a nonsingular quadric in $\left.\mathbb{P}^{2}\right)$ and $H^{2 k-2 n+j+2}\left(v_{0}^{-1}\left(x_{j}\right), \mathbb{C}\right)$ gives us a one-dimensional non-trivial local system when $2 k=n+1, n-1$. In view of (4.6), (4.10) and (4.9) we conclude that

$$
\mathrm{IC}\left(\mathcal{O}_{2^{n-1} 1^{3}}, \mathcal{E}_{n-1}\right)[-1] \oplus \operatorname{IC}\left(\mathcal{O}_{2^{n-1} 1^{3}}, \mathcal{E}_{n-1}\right)[1] \text { is a direct summand of } v_{*} \mathbb{C}[-] \text {. }
$$

This proves (4.12) for $i=\left[\frac{n}{2}\right]$. By induction hypothesis, suppose that (4.12) holds for all $j<i$. Let $2 k=(n-2 i)(n-2 i+1)+2 n-2 i-2$. Take the stalk at $x_{2 i}$ of $\mathcal{H}^{2 k-n^{2}-2 n+2}$ in (4.11), we get

$$
\operatorname{dim} \mathcal{H}_{x_{2 i}}^{2 k-n^{2}-2 n+2} \bigoplus_{a_{i}=0}^{k_{i}} \mathrm{IC}\left(\mathcal{O}_{2^{2 i} 1^{2 n+1-4 i}}, \mathcal{E}_{2 i}^{\oplus m_{a_{i}}^{i}}\right)\left[k_{i}-2 a_{i}\right]=1
$$

i.e. $\operatorname{dim} \mathcal{H}_{x_{2 i}}^{n-2 i-\operatorname{dim} \mathcal{O}_{2^{2 i} 1^{2 n+1-4 i}}} \bigoplus_{a_{i}=0}^{k_{i}} \operatorname{IC}\left(\mathcal{O}_{2^{2 i} 1^{2 n+1-4 i}}, \mathcal{E}_{2 i}^{\oplus m_{a_{i}}^{i}}\right)\left[k_{i}-2 a_{i}\right]=1$, 
here we use (4.9), (4.10) and the fact that

$$
\operatorname{dim} \mathcal{H}_{x_{2 i}}^{2 k-n^{2}-2 n+2} \bigoplus_{j=i+1}^{\left[\frac{n}{2}\right]} \bigoplus_{a_{j}=0}^{n-2 j} \operatorname{IC}\left(\mathcal{O}_{2^{2 j} 1^{2 n+1-4 j}}, \mathcal{E}_{2 j}^{\oplus m_{a_{j}}^{j}}\right)\left[n-2 j-2 a_{j}\right]=0
$$

as $2 k-n^{2}-2 n+2+2 j(2 n+1-2 j)+n-2 j-2 a_{j} \geq 4(j-i)(n+1-i-j)>0$. Thus we can conclude now that $m_{n-2 i}^{i}=1$ which implies $m_{0}^{i}=1$. This completes the proof of (4.12).

Comparing with (4.4), we conclude that

$$
\mathfrak{F}\left(\mathrm{IC}\left(\mathcal{O}_{2^{2 i} 1^{2 n+1-4 i}}, \mathcal{E}_{2 i}\right)\right)=\mathrm{IC}\left(\mathfrak{g}_{1}, \mathcal{F}_{2 i}\right)
$$

and $m_{a_{i}}^{i}=1$ for all $0 \leq a_{i} \leq n-2 i$. This finishes the proof of Theorem 2.3,

Finally taking Fourier transform of (4.11) we obtain the decomposition in (4.5).

Corollary 4.1. (1) We have $\mathcal{H}_{x_{j}}^{k} \mathrm{IC}\left(\mathcal{O}_{2^{i} 1^{2 n-2 i+1}}, \mathbb{C}\right)=0$ for $k$ odd and $j \leq i$.

(2) We have $\mathcal{H}_{x_{j}}^{k} \mathrm{IC}\left(\mathcal{O}_{2^{2 i} 1^{2 n-4 i+1}}, \mathcal{E}_{2 i}\right)=0$ for $k$ odd and $j \leq i$.

Proof. (1) follows from equation (4.7), the fact that $n^{2}+n$ is even and the fact that $H^{\text {odd }}\left(v_{0}^{-1}\left(x_{j}\right), \mathbb{C}\right)=0$. Taking $\mathcal{H}_{x_{j}}^{k}$ on both sides of the equation (4.5), we see that (2) follows from the fact that $n^{2}+n$ is even and the fact that $H^{\text {odd }}\left(v^{-1}\left(x_{j}\right), \mathbb{C}\right)=0$.

Remark 4.2. For $x \in \mathfrak{g}_{1}^{r s}$, the fibers

$$
Z_{x}:=\check{v}_{2}^{-1}(x)=\left\{\left(0 \subset V_{n} \subset V_{n}^{\perp} \subset V\right) \mid \operatorname{rank}\left(\bar{x}: V_{n} \rightarrow V / V_{n}^{\perp}\right) \leq 1\right\}
$$

are the over-generalized Kummer varieties introduced in [Re, p.80]. For example, when $n=2$, the map $f_{x}: F_{x} \rightarrow Z_{x}$ in (4.1) realizes $Z_{x}$ as the quotient $F_{x} / \sigma$ of the Fano variety $F_{x}$ of lines in the complete intersection $\tilde{Q} \cap \tilde{Q}_{x}$ of 2 quadrics in $\mathbb{P}^{5}$ by the involution $\sigma$ (see \$3.2). There are 16 fixed points of $\sigma$ on $F_{x}$ corresponding to 16 singular points of $Z_{x} \simeq F_{x} / \sigma$. The Hessenberg variety $H_{x}:=\check{v}^{-1}(x)$ is the blow up of $Z_{x}$ at those singular points, which is isomorphic to the Kummer K3 surface coming from the $\operatorname{Jac}\left(C_{x}\right)$-torsor $F_{x}$ together with the involution $\sigma$.

4.2. Proof of Theorem 2.2. Let us start with the matching for the $\operatorname{IC}\left(\mathcal{O}_{2^{i} 1^{2 n-2 i+1}}, \mathbb{C}\right)^{\prime}$ 's with $i$ odd.

Proposition 4.3. If $i$ is odd, then $\mathfrak{F}\left(\mathrm{IC}\left(\mathcal{O}_{2^{i} 1^{2 n-2 i+1}}, \mathbb{C}\right)\right)=\operatorname{IC}\left(\mathfrak{g}_{1}, \mathcal{L}_{i}\right)$.

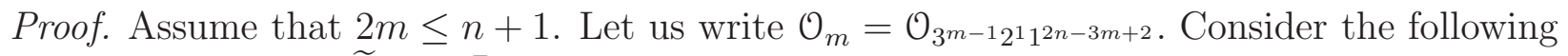
resolution map $\tau_{m}: \widetilde{\mathcal{O}}_{m} \rightarrow \overline{\mathcal{O}}_{m}$, where

$$
\widetilde{\mathcal{O}}_{m}=\left\{\left(x, 0 \subset V_{m-1} \subset V_{m} \subset V_{m}^{\perp} \subset V_{m-1}^{\perp} \subset V=\mathbb{C}^{2 n+1}\right) \mid x \in \mathfrak{g}_{1}, x V_{m}=0, x V_{m}^{\perp} \subset V_{m-1}\right\} .
$$

We show that

$$
\mathrm{IC}\left(\mathcal{O}_{2^{2 m-1}} 1^{2 n-4 m+3}, \mathbb{C}\right) \text { is a direct summand of } \tau_{m *} \mathbb{C}[-] \text {. }
$$

Recall that $\mathcal{O}_{\lambda} \subset \overline{\mathcal{O}}_{\mu}$ if and only if $\mu \geq \lambda$ if and only if $\lambda^{t} \geq \mu^{t}$. Thus we have

$$
\overline{\mathcal{O}}_{3^{i} 2^{j} 1^{2 n+1-3 i-2 j}} \supset \mathcal{O}_{3^{i^{\prime}} 2^{j^{\prime}} 1^{2 n+1-3 i^{\prime}-2 j^{\prime}}} \text { if and only if } i \geq i^{\prime}, 2 i+j \geq 2 i^{\prime}+j^{\prime} .
$$


In particular, $\mathcal{O}_{2^{2 m-1} 1^{2 n-4 m+3}} \subset \overline{\mathcal{O}}_{3^{i} 2^{j} 1^{2 n+1-3 i+2 j}} \subset \overline{\mathcal{O}}_{m}$ if and only if $i \leq m-1$ and $2 i+j=$ $2 m-1$.

We show in [CVX1, Lemma 2.4], independently of this paper, that for $i \in[0, m-1]$ and $x_{i} \in \mathcal{O}_{3^{i} 2^{2 m-1-2 i} 1^{2 n-4 m+3+i}}, \tau_{m}^{-1}\left(x_{i}\right) \cong \mathrm{OGr}(m-1-i, 2 m-1-2 i)$. It is then easy to check that

$$
2 \operatorname{dim} \tau_{m}^{-1}\left(x_{i}\right)=\operatorname{codim}_{\overline{\mathcal{O}}_{m}} \mathcal{O}_{x_{i}}=(m-i-1)(m-i) .
$$

Thus (4.13) follows from the discussion above, (4.14) and the decomposition theorem.

Consider the map

$$
\check{\tau}_{m}:\left\{\left(x, 0 \subset V_{m-1} \subset V_{m} \subset V_{m}^{\perp} \subset V_{m-1}^{\perp} \subset \mathbb{C}^{2 n+1}\right) \mid x \in \mathfrak{g}_{1}, x V_{m-1} \subset V_{m}, x V_{m} \subset V_{m}^{\perp}\right\} \rightarrow \mathfrak{g}_{1} .
$$

We have that

$$
\mathfrak{F}\left(\tau_{m *} \mathbb{C}[-]\right) \cong \check{\tau}_{m *} \mathbb{C}[-] .
$$

By Proposition 3.1, $\mathfrak{F}\left(\mathrm{IC}\left(\mathcal{O}_{2^{2 m-1}} 1^{2 n-4 m+3}, \mathbb{C}\right)\right)=\mathrm{IC}\left(\mathfrak{g}_{1}, \mathcal{L}_{i}\right)$ for some $i \in[1, n]$. We show in CVX1, Remark 4.9], again independently of this paper, that among the $\operatorname{IC}\left(\mathfrak{g}_{1}, \mathcal{L}_{i}\right)$ 's $(i \geq 1)$, only $\operatorname{IC}\left(\mathfrak{g}_{1}, \mathcal{L}_{2 j-1}\right), 1 \leq j \leq m$, appear in the decomposition of $\check{\tau}_{m *} \mathbb{C}[-]$. Thus by induction on $m$, (4.13) and (4.15) imply that

$$
\mathfrak{F}\left(\mathrm{IC}\left(\mathcal{O}_{2^{2 m-1} 1^{2 n-4 m+3}}, \mathbb{C}\right)\right)=\operatorname{IC}\left(\mathfrak{g}_{1}, \mathcal{L}_{2 m-1}\right) .
$$

So Theorem 2.2 is now reduced to the statement that for even $i$,

$$
\mathfrak{F}\left(\mathrm{IC}\left(\mathcal{O}_{2^{i} 1^{2 n-2 i+1}}, \mathbb{C}\right)\right)=\operatorname{IC}\left(\mathfrak{g}_{1}, \mathcal{L}_{i}\right) .
$$

For this we observe that $\operatorname{dim} \mathcal{L}_{i} \neq \operatorname{dim} \mathcal{L}_{j}$ for $i \neq j$, hence it suffices to show that the generic rank of $\mathfrak{F}\left(\mathrm{IC}\left(\mathcal{O}_{2^{i} 1^{2 n-2 i+1}}, \mathbb{C}\right)\right)$ is equal to $\operatorname{dim} \mathcal{L}_{i}$.

We make use of the theory of characteristic cycles to compute the generic rank of $\mathfrak{F}\left(\mathrm{IC}\left(\mathcal{O}_{2^{i} 1^{2 n-2 i+1}}, \mathbb{C}\right)\right)$. Recall the following facts about characteristic cycles (see $\left.[\mathrm{KS}]\right)$ :

(1) The Fourier transform preserves the characteristic cycle of conic sheaves.

(2) Let $\mathcal{F}$ be an irreducible perverse sheave on $\mathfrak{g}_{1}$ and let $r$ be the generic rank of $\mathcal{F}$. Then the characteristic cycle of $\mathcal{F}$, denoted by $\mathrm{CC}(\mathcal{F})$, satisfies

$$
\mathrm{CC}(\mathcal{F})=r \cdot\left(T_{\mathfrak{g}_{1}}^{*} \mathfrak{g}_{1}\right)+\cdots
$$

We will prove the following equality of characteristic cycles.

Proposition 4.4. Assume that $i$ is even. We have

$$
\mathrm{CC}\left(\mathrm{IC}\left(\mathcal{O}_{2^{i} 1^{2 n-2 i+1}}, \mathbb{C}\right)\right)=\mathrm{CC}\left(\mathrm{IC}\left(\mathcal{O}_{2^{i} 1^{2 n-2 i+1}}, \mathcal{E}_{i}\right)\right)+\mathrm{CC}\left(\mathrm{IC}\left(\mathcal{O}_{2^{i-1} 1^{2 n-2 i+3}}, \mathbb{C}\right)\right) .
$$

In Theorem 2.3 we have shown that for even $i$, the generic rank of $\mathfrak{F}\left(\operatorname{IC}\left(\mathcal{O}_{2^{i} 1^{2 n-2 i+1}}, \mathcal{E}_{i}\right)\right)$ is $\operatorname{dim} \mathcal{F}_{i}$. This together with the equality above imply Theorem 2.2. Indeed, using Propositions 4.3 and 4.4 we see that

$$
\begin{gathered}
\mathrm{CC}\left(\mathfrak{F}\left(\mathrm{IC}\left(\mathcal{O}_{2^{i} 1^{2 n-2 i+1}}, \mathbb{C}\right)\right)\right)=\mathrm{CC}\left(\mathfrak{F}\left(\operatorname{IC}\left(\mathcal{O}_{2^{i} 1^{2 n-2 i+1}}, \mathcal{E}_{i}\right)\right)\right)+\mathrm{CC}\left(\mathfrak{F}\left(\operatorname{IC}\left(\mathcal{O}_{2^{i-1} 1^{2 n-2 i+3}}, \mathbb{C}\right)\right)\right) \\
=\mathrm{CC}\left(\operatorname{IC}\left(\mathfrak{g}_{1}, \mathcal{F}_{i}\right)\right)+\mathrm{CC}\left(\operatorname{IC}\left(\mathfrak{g}_{1}, \mathcal{L}_{i-1}\right)\right)=r \cdot\left(T_{\mathfrak{g}_{1}}^{*} \mathfrak{g}_{1}\right)+\cdots
\end{gathered}
$$


where

$$
r=\operatorname{dim} \mathcal{F}_{i}+\operatorname{dim} \mathcal{L}_{i-1}=\left(\begin{array}{c}
2 n \\
i
\end{array}\right)-\left(\begin{array}{c}
2 n \\
i-2
\end{array}\right)+\left(\begin{array}{c}
2 n+1 \\
i-1
\end{array}\right)=\left(\begin{array}{c}
2 n+1 \\
i
\end{array}\right)=\operatorname{dim} \mathcal{L}_{i} .
$$

Hence the generic rank of $\mathfrak{F}\left(\operatorname{IC}\left(\mathcal{O}_{2^{i} 1^{2 n-2 i+1}}, \mathbb{C}\right)\right)$ is equal to $\operatorname{dim} \mathcal{L}_{i}$, and the theorem follows.

It remains to prove Proposition 4.4. The proof is given in the next subsection.

4.3. Proof of Proposition 4.4. Recall that for any variety $X$ and $\mathcal{F} \in D(X)$ we can consider the corresponding local Euler characteristic function

$$
\chi(\mathcal{F}): X \rightarrow \mathbb{Z}
$$

defined by

We have the following fact

$$
\chi(\mathcal{F})_{x}=\sum(-1)^{i} \operatorname{dim}\left(\mathcal{H}_{x}^{i} \mathcal{F}\right)
$$

Fact $4.5\left([\mathrm{KS}]\right.$, Theorem 9.7.11). Let $\mathcal{F}_{1}, \mathcal{F}_{2} \in D(X)$. Then $\mathrm{CC}\left(\mathcal{F}_{1}\right)=\mathrm{CC}\left(\mathcal{F}_{2}\right)$ if and only if $\chi\left(\mathcal{F}_{1}\right)=\chi\left(\mathcal{F}_{2}\right)$.

By the fact above we are reduced to show the following:

Proposition 4.6. For even $i$, we have

$$
\chi\left(\mathrm{IC}\left(\mathcal{O}_{2^{i} 1^{2 n-2 i}}, \mathbb{C}\right)\right)=\chi\left(\mathrm{IC}\left(\mathcal{O}_{2^{i} 1^{2 n-2 i}}, \mathcal{E}_{i}\right)\right)+\chi\left(\mathrm{IC}\left(\mathcal{O}_{2^{i-1} 1^{2 n-2 i+2}}, \mathbb{C}\right)\right) .
$$

In $\$ 5$ Theorem 5.1 and Theorem 5.2 , we show that

$$
\begin{gathered}
\chi\left(\mathrm{IC}\left(\mathcal{O}_{2^{i} 1^{2 n-2 i+1}}, \mathbb{C}\right)\right)=\chi\left(\operatorname{IC}\left(\mathcal{O}_{2^{i} 1^{2 n-2 i}}^{\prime}, \mathbb{C}\right)\right) \text { for all } i \\
\chi\left(\operatorname{IC}\left(\mathcal{O}_{2^{i} 1^{2 n-2 i+1}}, \mathcal{E}_{i}\right)\right)=\chi\left(\operatorname{IC}\left(\mathcal{O}_{2^{i} 1^{2 n-2 i}}^{\prime}, \mathcal{E}_{i}^{\prime}\right)\right) \text { for even } i,
\end{gathered}
$$

where $\mathcal{O}_{2^{i} 1^{2 n-2 i}}^{\prime}$ is the nilpotent $S p(2 n)$-orbit in $\mathfrak{s p}(2 n)$ corresponding to the partition $2^{i} 1^{2 n-2 i}$ and $\mathcal{E}_{i}^{\prime}$ is the unique non-trivial irreducible $S p(2 n)$-equivariant local system on $\mathcal{O}_{2^{i} 1^{2 n-2 i}}^{\prime}$.

As such, we prove the proposition above making use of the classical Springer correspondence for symplectic group $S p(2 n)$. As discussed earlier, this will complete the proof of Theorem 2.2.

Proof of Proposition 4.6. The proof is reduced to proving the following: for even $i, j \leq i$, and $x_{j}^{\prime} \in \mathcal{O}_{2^{j} 1^{2 n-2 j}}^{\prime}$ we have

$$
\chi\left(\mathrm{IC}\left(\mathcal{O}_{2^{i} 1^{2 n-2 i}}^{\prime}, \mathbb{C}\right)\right)_{x_{j}^{\prime}}=\chi\left(\mathrm{IC}\left(\mathcal{O}_{2^{i} 1^{2 n-2 i}}^{\prime}, \mathcal{E}_{i}^{\prime}\right)\right)_{x_{j}^{\prime}}+\chi\left(\mathrm{IC}\left(\mathcal{O}_{2^{i-1} 1^{2 n-2 i+2}}^{\prime}, \mathbb{C}\right)\right)_{x_{j}^{\prime}}
$$

We will show that

$$
\begin{aligned}
& \chi\left(\mathrm{IC}\left(\mathcal{O}_{2^{i} 1^{2 n-2 i}}^{\prime}, \mathbb{C}\right)\right)_{x_{j}^{\prime}}=\chi\left(\mathrm{IC}\left(\mathcal{O}_{2^{i-j} 1^{2 n-2 i}}^{\prime}, \mathbb{C}\right)\right)_{0} \\
& \chi\left(\mathrm{IC}\left(\mathcal{O}_{2^{i-j} 1^{2 n-2 i}}^{\prime}, \mathbb{C}\right)\right)_{0}=\left(\begin{array}{c}
n-j \\
{\left[\frac{i-j}{2}\right]}
\end{array}\right) \\
& \chi\left(\mathrm{IC}\left(\mathcal{O}_{2^{2 i} 1^{2 n-4 i}}^{\prime}, \mathcal{E}_{2 i}\right)\right)_{x_{j}^{\prime}}=\left(\begin{array}{c}
n-j \\
i-\frac{j}{2}
\end{array}\right)-\left(\begin{array}{c}
n-j \\
i-\frac{j}{2}-1
\end{array}\right) \text { if } j \text { is even, } \\
& \chi\left(\mathrm{IC}\left(\mathcal{O}_{2^{2 i} 1^{2 n-4 i}}^{\prime}, \mathcal{E}_{2 i}^{\prime}\right)\right)_{x_{j}^{\prime}}=0 \text { if } j \text { is odd }
\end{aligned}
$$


The equality (4.18) follows.

Proof of (4.19). The equality (4.19) follows from (4.16) and Proposition 5.3 (see \$5), which states that $\mathcal{H}_{x}^{k} \mathrm{IC}\left(\mathcal{O}_{2^{i} 1^{2 n-2 i+1}}, \mathbb{C}\right)=\mathcal{H}_{0}^{k+s_{j}} \mathrm{IC}\left(\mathcal{O}_{2^{i-j} 1^{2 n-2 i+1}}, \mathbb{C}\right), s_{j}=j(2 n+1-j)$. Indeed, since $s_{j}$ is even, we have

$$
\begin{gathered}
\chi\left(\mathrm{IC}\left(\mathcal{O}_{2^{i} 1^{2 n-2 i}}^{\prime}, \mathbb{C}\right)\right)_{x_{j}^{\prime}}=\sum(-1)^{k} \operatorname{dim} \mathcal{H}_{x_{j}^{\prime}}^{k} \mathrm{IC}\left(\mathcal{O}_{2^{i} 1^{2 n-2 i}}^{\prime}, \mathbb{C}\right) \\
=\sum(-1)^{k+s_{j}} \operatorname{dim} \mathcal{H}_{0}^{k+s_{j}} \mathrm{IC}\left(\mathcal{O}_{2^{i-j} 1^{2 n-2 i}}^{\prime}, \mathbb{C}\right)=\chi\left(\mathrm{IC}\left(\mathcal{O}_{2^{i-j} 1^{2 n-2 i}}^{\prime}, \mathbb{C}\right)\right)_{0}
\end{gathered}
$$

Proof of (4.20) and (4.21). We work in the classical Springer correspondence setting for $\mathfrak{s p}(2 n)$. Let $\mathcal{N}=\mathcal{N}_{\mathfrak{s p}(2 n)}$ and $G=S p(2 n)$ in this proof. Recall that we have the Springer resolution $\varphi: \widetilde{\mathcal{N}} \rightarrow \mathcal{N}$ and

$$
\varphi_{*} \mathbb{C}[-] \cong \bigoplus_{(\mathcal{O}, \mathcal{\varepsilon})} \mathrm{IC}(\mathcal{O}, \mathcal{E}) \otimes V_{\mathcal{O}, \mathcal{\varepsilon}}
$$

Here $(\mathcal{O}, \mathcal{E})$ runs through the pairs consisting of a nilpotent $G$-orbit $\mathcal{O} \subset \mathcal{N}$ and an irreducible $G$-equivarant local system on $\mathcal{O}$, that appear in the Springer correspondence. Moreover $V_{\mathcal{O}, \mathcal{E}}$ denotes the irreducible Weyl group representation corresponding to the pair $(\mathcal{O}, \mathcal{E})$ under Springer correspondence. It follows that

$$
\chi(\mathrm{IC}(\mathcal{O}, \mathcal{E}))_{x}=\left[V_{\mathcal{O}, \mathcal{\varepsilon}}: H^{*}\left(\mathcal{B}_{x}, \mathbb{C}\right)\right]
$$

for $x \in \overline{\mathcal{O}}$. Here $\mathcal{B}_{x}=\varphi^{-1}(x)$ is the Springer fiber and $\left[V_{\mathcal{O}, \varepsilon}: H^{*}\left(\mathcal{B}_{x}, \mathbb{C}\right)\right]$ denotes the multiplicity of $V_{\mathcal{O}, \varepsilon}$ in the Weyl group representation $H^{*}\left(\mathcal{B}_{x}, \mathbb{C}\right)$.

Let us denote the Weyl group of type $C_{n}$ by $W_{n}$. It is well known that the irreducible representations of $W_{n}$ are parametrized by pairs of partitions $(\alpha)(\beta)$ such that $|\alpha|+|\beta|=n$. Lusztig in [L1] has computed the (generalized) Springer correspondence explicitly. In our case, we have

$$
\begin{aligned}
& \left.V_{\left(\mathcal{O}_{22 i 12 n-4 i}^{\prime}, \mathbb{C}\right)}=\left(1^{i}\right)\left(1^{n-i}\right), V_{\left(\mathcal{O}_{22 i-1}^{\prime} 2 n-4 i+2\right.}, \mathbb{C}\right)=\left(1^{n-i+1}\right)\left(1^{i-1}\right), \\
& \left.V_{\left(\mathcal{O}_{22 i}^{\prime} 2 n-4 i\right.}, \mathcal{E}_{2 i}^{\prime}\right)=(0)\left(2^{i} 1^{n-2 i}\right) \text {, }
\end{aligned}
$$

and $\mathrm{IC}\left(\mathcal{O}_{2^{2 i-1} 1^{2 n-4 i+2}}^{\prime}, \mathcal{E}_{2 i-1}^{\prime}\right)$ does not appear in the decomposition of $\varphi_{*} \mathbb{C}[-]$. It follows from (4.24) and (4.25) that

$$
\chi\left(\mathrm{IC}\left(\mathcal{O}_{2^{i} 1^{2 n-2 i}}^{\prime}, \mathbb{C}\right)\right)_{0}=\left[V_{\left(\mathcal{O}_{2^{i} 1^{2 n-2 i}}^{\prime}, \mathbb{C}\right)}: H^{*}(\mathcal{B}, \mathbb{C})\right]=\operatorname{dim} V_{\left(\mathcal{O}_{2^{i} 1^{2 n-2 i}}^{\prime}, \mathbb{C}\right)}=\left(\begin{array}{c}
n \\
{\left[\frac{i}{2}\right]}
\end{array}\right) .
$$

This proves (4.20).

We prove (4.21). Recall that $H^{\text {odd }}\left(\mathcal{B}_{x}, \mathbb{C}\right)=0$ (see [CLP]) and if $x \in$ Lie $L$ is regular nilpotent, we have (see [L3])

$$
\sum H^{2 k}\left(\mathcal{B}_{x}, \mathbb{C}\right)=\operatorname{Ind}_{W_{L}}^{W_{n}} \mathbb{C},
$$

where $L$ is a Levi subgroup of $G$ and $W_{L}$ is the Weyl group of $L$. 
Consider our elements $x_{j}^{\prime} \in \mathcal{O}_{2^{j}{ }^{2 n-2 j}}^{\prime}$, where $j \leq 2 i$. Assume that $j=2 j_{0}$. We can find a Levi subgroup $L \subset S p(2 n)$ such that $L \cong G L(2) \times \cdots \times G L(2)\left(j_{0}\right.$ copies $) \subset L_{0}=G L(n)$, and $x_{j}^{\prime} \in$ Lie $L$ is regular, where $L_{0}$ is a Levi subgroup of a maximal parabolic subgroup. We have $W_{L} \cong S_{2} \times \cdots \times S_{2}\left(j_{0}\right.$ copies $) \subset S_{n}$. Thus

$$
\begin{aligned}
& \left.\chi\left(\operatorname{IC}\left(\mathcal{O}_{2^{2 i} 1^{2 n-4 i}}^{\prime}, \mathcal{E}_{2 i}^{\prime}\right)\right)_{x_{j}^{\prime}} \stackrel{[4.24)}{=}\left[V_{\left(\mathcal{O}_{22 i}^{\prime} 2 n-4 i\right.}, \varepsilon_{2 i}^{\prime}\right), H^{*}\left(\mathcal{B}_{x_{j}^{\prime}}, \mathbb{C}\right)\right] \stackrel{[4.26)}{=}\left[(0)\left(2^{i} 1^{n-2 i}\right), \operatorname{Ind}_{W_{L}}^{W_{n}} \mathbb{C}\right] \\
& =\left[(0)\left(2^{i} 1^{n-2 i}\right), \operatorname{Ind}_{S_{n}}^{W_{n}} \operatorname{Ind}_{W_{L}}^{S_{n}} \mathbb{C}\right]=\left[\operatorname{Res}_{S_{n}}^{W_{n}}(0)\left(2^{i} 1^{n-2 i}\right), \operatorname{Ind}_{W_{L}}^{S_{n}} \mathbb{C}\right]_{S_{n}} \\
& =\left[\left(2^{i} 1^{n-2 i}\right), \operatorname{Ind}_{W_{L}}^{S_{n}} \mathbb{C}\right]_{S_{n}}=\left[2^{i} 1^{n-2 i}, H^{*}\left(\mathcal{B}_{2^{j_{0}} 1^{n-2 j_{0}}}^{G L(n)}, \mathbb{C}\right)\right]_{S_{n}}=\chi\left(\mathrm{IC}\left(\mathcal{O}_{2^{i} 1^{n-2 i}}, \mathbb{C}\right)\right)_{2^{j_{0}} 1^{n-2 j_{0}}}^{G L(n)} .
\end{aligned}
$$

Here in the last two equalities, $\mathcal{B}_{2^{j 0} 1^{n-2 j_{0}}}^{G L(n)}$ denotes the Springer fiber of an element in the nilpotent orbit corresponding to the partition $2^{j_{0}} 1^{n-2 j_{0}}$ in $\mathfrak{g l}(n)$, and $\chi\left(\operatorname{IC}\left(\mathcal{O}_{2^{i} 1^{n-2 i}}, \mathbb{C}\right)\right)_{2^{j_{0}} 1^{n-2 j_{0}}}^{G L(n)}$ is defined analogously in $G L(n)$. Now it follows from the classical result for $G L(n)$ that

$$
\chi\left(\mathrm{IC}\left(\mathcal{O}_{2^{i} 1^{n-2 i}}, \mathbb{C}\right)\right)_{2^{j_{0}} 1^{n-2 j_{0}}}^{G L(n)}=K_{2^{i} 1^{n-2 i}, 2^{j_{0}} 1^{n-2 j_{0}}}=K_{2^{i-j_{0} 1^{n-2 i}, 1^{n-2 j_{0}}}}=\left(\begin{array}{c}
n-2 j_{0} \\
i-j_{0}
\end{array}\right)-\left(\begin{array}{c}
n-2 j_{0} \\
i-j_{0}-1
\end{array}\right),
$$

where $K_{\lambda, \mu}$ denotes the Kostka number. This proves (4.21).

Proof of (4.22). Let $x_{j} \in \mathcal{O}_{2^{j} 1^{2 n-2 j+1}}$, where $j$ is odd. In view of (4.17), it suffices to show that $\chi\left(\operatorname{IC}\left(\mathcal{O}_{2^{2 i} 1^{2 n-4 i+1}}, \mathcal{E}_{2 i}\right)\right)_{x_{j}}=0$. We prove this using the decomposition in (4.5) (see $\$ 4.1$ )

$$
\begin{aligned}
& v_{*} \mathbb{C}\left[n^{2}+2 n-2\right] \cong \bigoplus_{a=0}^{n-2} \bigoplus_{i=0}^{n} \operatorname{IC}\left(\mathcal{O}_{2^{i} 1^{2 n-2 i+1}}, \mathbb{C}\right)[n-2-2 a] \\
& \oplus \bigoplus_{i=1}^{\left[\frac{n}{2}\right]} \bigoplus_{a_{i}=0}^{n-2 i} \operatorname{IC}\left(\mathcal{O}_{2^{2 i} 1^{2 n+1-4 i}}, \mathcal{E}_{2 i}\right)\left[n-2 i-2 a_{i}\right] \oplus \bigoplus_{a=0}^{n} \operatorname{IC}\left(\mathcal{O}_{1^{2 n+1}}, \mathbb{C}\right)[n-2 a] .
\end{aligned}
$$

Note that $\mathcal{H}_{x_{j}}^{\text {odd }} \operatorname{IC}\left(\mathcal{O}_{2^{i} 1^{2 n+1-2 i}}, \mathbb{C}\right)=\mathcal{H}_{x_{j}}^{\text {odd }} \operatorname{IC}\left(\mathcal{O}_{2^{2 i} 1^{2 n+1-4 i}}, \mathcal{E}_{2 i}\right)=0$ (see Corollary 4.1). Thus $\chi\left(\mathrm{IC}\left(\mathcal{O}_{2^{2 i} 1^{2 n+1-4 i}}, \mathcal{E}_{2 i}\right)\right)_{x_{j}} \geq 0$. It follows from the decomposition above that

$$
\chi\left(H^{*}\left(v^{-1}\left(x_{j}\right), \mathbb{C}\right)=(n-1) \sum_{i=j}^{n} \chi\left(\operatorname{IC}\left(\mathcal{O}_{2^{i} 1^{2 n+1-2 i}}, \mathbb{C}\right)\right)_{x_{j}}+\sum_{j \leq 2 i \leq n} \chi\left(\operatorname{IC}\left(\mathcal{O}_{22^{2 i} 1^{2 n+1-4 i}}, \mathcal{E}_{2 i}\right)\right)_{x_{j}} .\right.
$$

We have $\chi\left(H^{*}\left(v^{-1}\left(x_{j}\right), \mathbb{C}\right)=(n-1) 2^{n-j}\right.$ (here $v^{-1}\left(x_{j}\right)$ is a quadric bundle over $\operatorname{OGr}(n-$ $j, 2 n-2 j+1$ ) with fibers having euler characteristic $n-1$, see \$4.1). Thus equations (4.16) and (4.20) imply that

$$
\sum_{i=j}^{n} \chi\left(\operatorname{IC}\left(\mathcal{O}_{2^{i} 1^{2 n+1-2 i}}, \mathbb{C}\right)\right)_{x_{j}}=\sum_{i=j}^{n}\left(\begin{array}{c}
n-j \\
{\left[\frac{i-j}{2}\right]}
\end{array}\right)=2^{n-j} .
$$

It follows that $\chi\left(\operatorname{IC}\left(\mathcal{O}_{2^{2 i} 1^{2 n+1-4 i}}, \mathcal{E}_{2 i}\right)\right)_{x_{j}}=0$.

The proof of Proposition 4.4 is complete. This completes the proof of Theorem 2.2. 


\section{Stalks of the IC Sheaves}

In this section we establish an interesting isomorphism between stalks of the IC sheaves supported on nilpotent orbits of order two and stalks of the IC sheaves supported on nilpotent orbits of order two in $\mathfrak{s p}(2 n)$. More precisely, let $\mathcal{N}_{\mathfrak{s p}(2 n)}$ denote the nilpotent cone of $\mathfrak{s p}(2 n)$. Let $\mathcal{O}_{2^{i} 1^{2 n-2 i}}^{\prime} \subset \mathcal{N}_{\mathfrak{s p}(2 n)}$ denote the nilpotent orbit corresponding to the partition $2^{i} 1^{2 n-2 i}$. We have $\operatorname{dim} \mathcal{O}_{2^{i} 1^{2 n-2 i}}^{\prime}=\operatorname{dim} \mathcal{O}_{2^{i} 1^{2 n-2 i+1}}=i(2 n+1-i)$, and for each $i \geq 1$, there exists a unique nontrivial irreducible $S p(2 n)$-equivariant local system $\mathcal{E}_{i}^{\prime}$ on $\mathcal{O}_{2^{i} 1^{2 n-2 i}}^{\prime}$.

We have the following theorems, which were used in the proof of Theorem 2.2 in $\$ 4$. We remark that the proofs of these theorems are independent of $\$ 4$.

Theorem 5.1. For $x \in \mathcal{O}_{2^{j} 1^{2 n-2 j+1}}$ and $x^{\prime} \in \mathcal{O}_{2^{j} 1^{2 n-2 j}}^{\prime}$, we have

$$
\mathcal{H}_{x}^{k} \mathrm{IC}\left(\mathcal{O}_{2^{i} 1^{2 n-2 i+1}}, \mathbb{C}\right) \simeq \mathcal{H}_{x^{\prime}}^{k} \mathrm{IC}\left(\mathcal{O}_{2^{i} 1^{2 n-2 i}}^{\prime}, \mathbb{C}\right)
$$

Theorem 5.2. Assume that $i$ is even. For $x \in \mathcal{O}_{2^{j} 1^{2 n-2 j+1}}$ and $x^{\prime} \in \mathcal{O}_{2^{j} 1^{2 n-2 j}}^{\prime}$, we have

$$
\mathcal{H}_{x}^{k} \mathrm{IC}\left(\mathcal{O}_{2^{i} 1^{2 n-2 i+1}}, \mathcal{E}_{i}\right) \simeq \mathcal{H}_{x^{\prime}}^{k} \mathrm{IC}\left(\mathcal{O}_{2^{i} 1^{2 n-2 i}}^{\prime}, \mathcal{E}_{i}^{\prime}\right) .
$$

The following proposition was used in the proof of (4.19).

Proposition 5.3. Let $j \leq i$ and set

$$
s_{j}=\operatorname{dim} \mathcal{O}_{2^{i} 1^{2 n-2 i+1}}-\operatorname{dim} \mathcal{O}_{2^{i-j} 1^{2 n-2 i+1}}=j(2 n+1-j) .
$$

Then for $x \in \mathcal{O}_{2^{j} 1^{2 n-2 j+1}}$, we have

$$
\mathcal{H}_{x}^{k} \mathrm{IC}\left(\mathcal{O}_{2^{i} 1^{2 n-2 i+1}}, \mathbb{C}\right)=\mathcal{H}_{0}^{k+s_{j}} \mathrm{IC}\left(\mathcal{O}_{2^{i-j} 1^{2 n-2 i+1}}, \mathbb{C}\right) .
$$

Remark 5.4. Note that, in general, the singularities for $\overline{\mathcal{O}}_{2^{i} 1^{2 n-2 i+1}}$ and $\overline{\mathcal{O}}_{2^{i} 1^{2 n-2 i}}^{\prime}$ are nonisomorphic. For example, the Euler obstruction $\operatorname{Eu}\left(0, \mathcal{O}_{\min }^{\prime}\right)$ for the minimal orbit $\mathcal{O}_{\min }^{\prime}:=$ $\mathcal{O}_{2^{1} 1^{2 n-2}}^{\prime}$ is zero (see [EM]). On the other hand, using

$$
\mathrm{CC}\left(\mathrm{IC}\left(\mathcal{O}_{\text {min }}, \mathbb{C}\right)\right)=\mathrm{CC}\left(\mathfrak{F}\left(\left(\operatorname{IC}\left(\mathcal{O}_{\text {min }}, \mathbb{C}\right)\right)\right)=\mathrm{CC}\left(\mathrm{IC}\left(\mathfrak{g}_{1}, \mathcal{L}_{1}\right)\right)\right.
$$

and $\operatorname{dim} \mathcal{L}_{1}=2 n+1$ (see Theorem 2.2), one can check that $\operatorname{Eu}\left(0, \mathcal{O}_{\text {min }}\right)=2 n$, where $\mathcal{O}_{\text {min }}:=\mathcal{O}_{2^{1} 1^{2 n-1}}$.

5.1. Resolutions. For the proof of Theorem 5.1 and Theorem 5.2 we need several preliminary steps. We begin with the construction of resolutions of $\overline{\mathcal{O}}_{2^{i} 1^{2 n+1-2 i}}$ and $\overline{\mathcal{O}}_{2^{i} 1^{2 n-2 i}}^{\prime}$.

For the orbit $\mathcal{O}_{2^{i} 1^{2 n+1-2 i}} \subset \mathcal{N}_{1}, 1 \leq i \leq n$, consider Reeder's resolution map of $\overline{\mathcal{O}}_{2^{i} 1^{2 n+1-2 i}}$,

$$
\sigma_{i}:\left\{\left(x, 0 \subset V_{i} \subset V_{i}^{\perp} \subset V=\mathbb{C}^{2 n+1}\right) \mid x \in \mathfrak{g}_{1}, x V \subset V_{i}\right\} \rightarrow \overline{\mathcal{O}}_{2^{i} 1^{2 n+1-2 i}} .
$$

For the orbit $\mathcal{O}_{2^{i} 1^{2 n-2 i}}^{\prime} \subset \mathcal{N}_{\mathfrak{s p}(2 n)}$, we have the following resolution map for $\overline{\mathcal{O}}_{2^{i} 1^{2 n-2 i}}^{\prime}$ ([He] $)$

$$
\tau_{i}:\left\{\left(x, 0 \subset U_{i} \subset U_{i}^{\perp} \subset U=\mathbb{C}^{2 n}\right) \mid x \in \mathfrak{s p}(2 n), x U \subset U_{i}\right\} \rightarrow \overline{\mathcal{O}}_{2^{i} 1^{2 n-i}}^{\prime} .
$$

Here $U$ is a $2 n$-dimensional vector space equipped with a non-degenerate symplectic form $\langle$,$\rangle such that \mathfrak{s p}(2 n)=\mathfrak{s p}(U,\langle\rangle),, \operatorname{dim} U_{i}=i$ and $U_{i}^{\perp}=\left\{u \in U \mid\left\langle u, U_{i}\right\rangle=0\right\}$. 
We show that for $x \in \mathcal{O}_{2^{j} 1^{2 n-2 j+1}}$ and $x^{\prime} \in \mathcal{O}_{2^{j} 1^{2 n-2 j}}^{\prime}, j \leq i$,

$$
\begin{aligned}
& \text { We have } \operatorname{dim} \sigma_{i}^{-1}(x)=\operatorname{dim} \tau_{i}^{-1}\left(x^{\prime}\right) \text {. Moreover, } H^{*}\left(\sigma_{i}^{-1}(x), \mathbb{C}\right) \simeq H^{*}\left(\tau_{i}^{-1}\left(x^{\prime}\right), \mathbb{C}\right) . \\
& \text { The action of } A(x):=Z_{K}(x) / Z_{K}(x)^{0}\left(\text { resp. } A^{\prime}\left(x^{\prime}\right):=Z_{S p(2 n)}\left(x^{\prime}\right) / Z_{S p(2 n)}\left(x^{\prime}\right)^{0}\right)
\end{aligned}
$$

$$
\text { on } H^{*}\left(\sigma_{i}^{-1}(x), \mathbb{C}\right)\left(\text { resp. } H^{*}\left(\tau_{i}^{-1}\left(x^{\prime}\right), \mathbb{C}\right)\right) \text { is trivial. }
$$

Let $s_{i j}=2 \operatorname{dim}\left(\sigma_{i}^{-1}\left(x_{j}\right)\right)-\operatorname{codim}_{\overline{\mathcal{O}}_{2^{i} 1^{2 n-2 i+1}}} \mathcal{O}_{2^{j} 1^{2 n-2 j+1}}=2(i-j)(n-i)$, where $x_{j} \in \mathcal{O}_{2^{j} 1^{2 n-2 j+1}}$. It follows from (5.1) and (5.2) that

Lemma 5.5. We have

$$
\begin{aligned}
& \left(\sigma_{i}\right)_{*} \mathbb{C}[-]=\bigoplus_{j=0}^{i} \bigoplus_{k=0}^{s_{i j}} \operatorname{IC}\left(\mathcal{O}_{2^{j} 1^{2 n-2 j+1}}, \mathbb{C}^{t^{i} k}\right)[ \pm k] \\
& \left(\tau_{i}\right)_{*} \mathbb{C}[-]=\bigoplus_{j=0}^{i} \bigoplus_{k=0}^{s_{i j}} \operatorname{IC}\left(\mathcal{O}_{2^{j}{ }^{2 n-2 j}}^{\prime}, \mathbb{C}^{\left(t_{j k}^{i}\right)^{\prime}}\right)[ \pm k]
\end{aligned}
$$

here $t_{i k}^{i}=\left(t_{i k}^{i}\right)^{\prime}=\delta_{k, 0}$.

We prove (5.1) and (5.2). For (5.1), we show that

$$
\sigma_{i}^{-1}(x) \cong \operatorname{OGr}(i-j, 2 n-2 j+1) \text { and } \tau_{i}^{-1}\left(x^{\prime}\right) \cong \operatorname{SpGr}(i-j, 2 n-2 j) .
$$

For (5.2), we show that the action of $A(x)$ (resp. $A^{\prime}\left(x^{\prime}\right)$ ) on $\sigma_{i}^{-1}(x)$ (resp. $\tau_{i}^{-1}\left(x^{\prime}\right)$ ) is trival, thus inducing a trivial action on $H^{*}\left(\sigma_{i}^{-1}(x), \mathbb{C}\right)\left(\right.$ resp. $\left.H^{*}\left(\tau_{i}^{-1}\left(x^{\prime}\right), \mathbb{C}\right)\right)$.

Let us consider the case of $x \in \mathcal{O}_{2^{j} 1^{2 n+1-2 j}}$ first. The following lemma allows us to choose convenient basis for $V$.

Lemma 5.6. Let $x \in \mathcal{O}_{\lambda} \subset \mathcal{N}_{1}$, where $\lambda=\left(\lambda_{1}, \lambda_{2}, \ldots, \lambda_{s}\right) \in \mathbf{P}(2 n+1)$. There exist $v_{i} \in V$, $i \in[1, s]$ such that $V=\operatorname{Span}\left\{x^{a_{i}} v_{i}, a_{i} \in\left[0, \lambda_{i}-1\right], i \in[1, s]\right\}$ and

$$
x^{\lambda_{i}} v_{i}=0,\left\langle x^{a_{i}} v_{i}, x^{b_{j}} v_{j}\right\rangle_{Q}=\delta_{i, j} \delta_{a_{i}+b_{j}, \lambda_{i}-1}, i, j \in[1, s] .
$$

Proof. We prove that the lemma holds also for even dimensional $V$ (the fact that some orbits when $\operatorname{dim} V$ is even are parametrized by the same partition does not affect the proof). We prove by induction on $\operatorname{dim} V$. It is clear when $\operatorname{dim} V=1$. Assume that $\operatorname{dim} V>1$. One checks easily that there exists $v \in V$ such that $\left\langle v, x^{\lambda_{1}-1} v\right\rangle_{Q} \neq 0$. We can assume that $\left\langle v, x^{\lambda_{1}-1} v\right\rangle_{Q}=1$. We have $x^{\lambda_{1}} v=0$ and $v, x v, \ldots, x^{\lambda_{1}-1} v$ are linearly independent. Let $W=\operatorname{Span}\left\{v, x v, \ldots, x^{\lambda_{1}-1} v\right\}$. Then $V=W \oplus W^{\perp}$ as $\left.\langle,\rangle_{Q}\right|_{W}$ is non-degenerate. Note that $W^{\perp}$ is $x$-stable and $\left.x\right|_{W^{\perp}} \in \mathcal{O}_{\lambda^{\prime}}$, where $\lambda^{\prime}=\left(\lambda_{2}, \ldots, \lambda_{s}\right)$. The lemma follows by induction.

Take a basis $\left\{v_{l}, x v_{l}, l \in[1, j], w_{s}, s \in[1,2 n+1-2 j]\right\}$ of $V$ as in Lemma [5.6. In terms of this basis, the fiber $\sigma_{i}^{-1}(x)$ can be described as follows. It consists of the flags $0 \subset V_{i} \subset V_{i}^{\perp} \subset$ $\mathbb{C}^{2 n+1}$, where $V_{i}=\operatorname{Span}\left\{x v_{k}, k \in[1, j]\right\} \oplus W_{i-j}$ and $W_{i-j} \subset \operatorname{Span}\left\{w_{s}, s \in[1,2 n+1-2 j]\right\}$ is such that $W_{i-j} \subset W_{i-j}^{\perp}$. Thus (5.5) holds in this case. Define $g \in Z_{K}(x)$ as follows. When $j$ is odd, let $g v_{k}=-v_{k}, g w_{s}=w_{s}$; when $j$ is even, let $g v_{1}=v_{2}, g v_{2}=v_{1}, g v_{k}=v_{k}, k \neq$ $1,2, g w_{s}=w_{s}$. (Note that the actions of $g$ on other basis vectors are determined by the 
property that $g \in Z_{K}(x)$.) It is easy to see that $g \notin Z_{K}(x)^{0}$. Thus we can identify $A(x)$ with $\{g, 1\}$. Now it is easy to see that $A(x)$ fixes each flag in $\sigma_{i}^{-1}(x)$, thus acting trivially.

The proof for the case of $x^{\prime} \in \mathcal{O}_{2^{j} 1^{2 n-2 j}}^{\prime}$ is entirely similar. There exist vectors $v_{1}^{\prime}, \ldots, v_{j}^{\prime}, w_{1}^{\prime}, \ldots, w_{n-j}^{\prime}, u_{1}^{\prime}, \ldots, u_{n-j}^{\prime} \in U=\mathbb{C}^{2 n}$ such that $x^{\prime 2} v_{l}^{\prime}=0, l \in[1, j], x^{\prime} w_{s}^{\prime}=$ $x^{\prime} u_{s}^{\prime}=0, s \in[1, n-j], U=\operatorname{Span}\left\{v_{l}^{\prime}, x^{\prime} v_{l}^{\prime}, l \in[1, j], w_{s}^{\prime}, u_{s}^{\prime}, s \in[1, n-j]\right\}$, and

$$
\left\langle v_{k}^{\prime}, v_{l}^{\prime}\right\rangle=\left\langle v_{k}^{\prime}, w_{s}^{\prime}\right\rangle=\left\langle v_{k}^{\prime}, u_{s}^{\prime}\right\rangle=\left\langle w_{s}^{\prime}, w_{t}^{\prime}\right\rangle=\left\langle u_{s}^{\prime}, u_{t}^{\prime}\right\rangle=0,\left\langle v_{k}^{\prime}, x^{\prime} v_{l}^{\prime}\right\rangle=\delta_{k, l},\left\langle w_{s}^{\prime}, u_{t}^{\prime}\right\rangle=\delta_{s, t} .
$$

(Note that $\left\langle x^{\prime} v, w\right\rangle=-\left\langle v, x^{\prime} w\right\rangle$ as $x^{\prime} \in \mathfrak{s p}(2 n)$.) In terms of this basis, the fiber $\tau_{i}^{-1}\left(x^{\prime}\right)$ can be described as follows. It consists of the flags $0 \subset U_{i} \subset U_{i}^{\perp} \subset \mathbb{C}^{2 n}$, where $U_{i}=$ $\operatorname{Span}\left\{x^{\prime} v_{k}^{\prime}, 1 \leq k \leq j\right\} \oplus W_{i-j}^{\prime}$ and $W_{i-j}^{\prime} \subset \operatorname{Span}\left\{w_{s}^{\prime}, u_{s}^{\prime}, 1 \leq s \leq n-j\right\}$ is such that $W_{i-j}^{\prime} \subset$ $W_{i-j}^{\prime \perp}$. Define $g^{\prime} \in Z_{S p(2 n)}\left(x^{\prime}\right)$ as follows. When $j$ is odd, let $g^{\prime} v_{k}^{\prime}=-v_{k}^{\prime}, g w_{s}^{\prime}=w_{s}^{\prime}, g u_{s}^{\prime}=u_{s}^{\prime}$; when $j$ is even, let $g^{\prime} v_{1}^{\prime}=v_{2}^{\prime}, g^{\prime} v_{2}^{\prime}=v_{1}^{\prime}, g^{\prime} v_{k}^{\prime}=v_{k}^{\prime}, k^{\prime} \neq 1,2, g^{\prime} w_{s}^{\prime}=w_{s}^{\prime}, g^{\prime} u_{s}^{\prime}=u_{s}^{\prime}$. As before we can identify $A^{\prime}\left(x^{\prime}\right)$ with $\left\{g^{\prime}, 1\right\}$ and it is easy to see that $A^{\prime}\left(x^{\prime}\right)$ fixes each flag in $\tau_{i}^{-1}\left(x^{\prime}\right)$, thus acting trivially.

5.2. The maps $\widetilde{\sigma}_{i}$ and $\widetilde{\tau}_{i}$. We preserve the notations from \$5.1. For the proof of Theorem 5.2, we need the following auxiliary maps.

For $\mathcal{O}_{2^{i} 1^{2 n-2 i+1}} \subset \mathcal{N}_{1}$, consider the map

$$
\tilde{\sigma}_{i}:\left\{\left(x, 0 \subset V_{i-1} \subset V_{i} \subset V_{i}^{\perp} \subset V_{i-1}^{\perp} \subset V\right) \mid x \in \mathfrak{g}_{1}, x V \subset V_{i}, x V_{i-1}^{\perp} \subset V_{i-1}\right\} \rightarrow \overline{\mathcal{O}}_{2^{i} 1^{2 n+1-2 i}} .
$$

For $\mathcal{O}_{2^{i} 1^{2 n-2 i}}^{\prime} \subset \mathcal{N}_{\mathfrak{s p}(2 n)}$, consider the following map

$\tilde{\tau}_{i}:\left\{\left(x^{\prime}, 0 \subset U_{i-1} \subset U_{i} \subset U_{i}^{\perp} \subset U_{i-1}^{\perp} \subset U\right) \mid x^{\prime} \in \mathfrak{s p}_{2 n}, x^{\prime} U \subset U_{i}, x^{\prime} U_{i-1}^{\perp} \subset U_{i-1}\right\} \rightarrow \overline{\mathcal{O}}_{2^{i} 1^{2 n-2 i}}^{\prime}$.

We show that for $x \in \mathcal{O}_{2^{j} 1^{2 n-2 j+1}}$ and $x^{\prime} \in \mathcal{O}_{2^{j} 1^{2 n-2 j}}^{\prime}, j \leq i$,

$$
\operatorname{dim} \tilde{\sigma}_{i}^{-1}(x)=\operatorname{dim} \tilde{\tau}_{i}^{-1}\left(x^{\prime}\right), H^{*}\left(\tilde{\sigma}_{i}^{-1}(x), \mathbb{C}\right) \simeq H^{*}\left(\tilde{\tau}_{i}^{-1}\left(x^{\prime}\right), \mathbb{C}\right), \text { and the latter }
$$

$$
\text { isomorphism is compatible with the actions of } A(x) \text { and } A^{\prime}\left(x^{\prime}\right) \text {. }
$$

It follows that

Lemma 5.7. We have

$$
\begin{aligned}
& \left(\tilde{\sigma}_{i}\right)_{*} \mathbb{C}[-]=\bigoplus_{j, k \geq 0} \operatorname{IC}\left(\mathcal{O}_{2^{j} 1^{2 n-2 j+1}}, \mathbb{C}^{m_{j k}^{i}}\right)[ \pm k] \bigoplus_{j \text { even, } k \geq 0} \operatorname{IC}\left(\mathcal{O}_{2^{j} 1^{2 n-2 j+1}}, \mathcal{E}_{j}^{a_{j k}^{i}}\right)[ \pm k] \\
& \left(\tilde{\tau}_{i}\right)_{*} \mathbb{C}[-]=\bigoplus_{j, k \geq 0} \operatorname{IC}\left(\mathcal{O}_{2^{j} 1^{2 n-2 j}}^{\prime}, \mathbb{C}^{\left(m_{j k}^{i}\right)^{\prime}}\right)[ \pm k] \bigoplus_{j \text { even, } k \geq 0} \operatorname{IC}\left(\mathcal{O}_{2^{j} 1^{2 n-2 j}}^{\prime},\left(\mathcal{E}_{j}^{\prime}\right)^{\left(a_{j k}^{i}\right)^{\prime}}\right)[ \pm k]
\end{aligned}
$$

We prove (5.6) and (5.7) in the reminder of this subsection.

The fiber $\widetilde{\sigma}_{i}^{-1}(x)$ is a quadric bundle over $\sigma_{i}^{-1}(x) \cong \operatorname{OGr}(i-j, 2 n-2 j+1)$ with fibers a quadric $\sum_{s=1}^{j} b_{s}^{2}=0$ in $\mathbb{P}^{i-1}=\left\{\left[b_{1}: b_{2}: \cdots: b_{i}\right]\right\}$. More precisely, we have an obvious map $\pi: \widetilde{\sigma}_{i}^{-1}(x) \rightarrow \sigma_{i}^{-1}(x)$,

$$
\left(0 \subset V_{i-1} \subset V_{i} \subset V_{i}^{\perp} \subset V_{i-1}^{\perp} \subset V\right):=\left(V_{i-1} \subset V_{i}\right) \mapsto\left(0 \subset V_{i} \subset V_{i}^{\perp} \subset V\right):=\left(V_{i}\right)
$$


by forgetting $V_{i-1}$. Now we describe the fibers of $\pi$. Recall that if $\left(V_{i}\right) \in \sigma_{i}^{-1}(x)$, then there exists $W_{i-j} \subset \operatorname{Span}\left\{w_{k}, k \in[1,2 n+1-2 j]\right\}$ with $W_{i-j} \subset W_{i-j}^{\perp}$ such that $V_{i}=\operatorname{Span}\left\{x v_{k}, k \in\right.$ $[1, j]\} \oplus W_{i-j}$. Let $\left[b_{1}: b_{2}: \cdots: b_{i}\right]$ be the homogenous coordinates of $\mathbb{P}\left(V_{i}\right)$ given by the basis $\left\{x v_{k}, k \in[1, j], \tilde{w}_{l}, l \in[1, i-j]\right\}$ of $V_{i}$, where $\left\{\tilde{w}_{l}\right\}$ is a basis of $W_{i-j}$. It is easy to check that the fibers of $\pi$ are isomorphic to the quadric $Q: \sum_{s=1}^{j} b_{s}^{2}=0$ in $\mathbb{P}\left(V_{i}\right) \cong \mathbb{P}^{i-1}$. It follows that

$$
H^{*}\left(\widetilde{\sigma}_{i}^{-1}(x), \mathbb{C}\right) \cong H^{*}(Q, \mathbb{C}) \otimes H^{*}\left(\sigma_{i}^{-1}(x), \mathbb{C}\right) .
$$

We describe the action of $A(x)$ on $H^{*}\left(\widetilde{\sigma}_{i}^{-1}(x), \mathbb{C}\right)$. As we have shown that $A(x)$ acts trivially on $H^{*}\left(\sigma_{i}^{-1}(x), \mathbb{C}\right)$, it suffices to describe the action of $A(x)$ on $H^{*}(Q, \mathbb{C})$.

We claim that if $j$ is odd, then $A(x)$ acts on $H^{*}(Q, \mathbb{C})$ trivially, thus acting trivially on $H^{*}\left(\widetilde{\sigma}_{i}^{-1}(x), \mathbb{C}\right)$, and if $j$ is even, then $A(x)$ acts on $H^{2 k}(Q, \mathbb{C})$ trivially if $2 k \neq 2 i-j-2$ and $H^{2 i-j-2}(Q, \mathbb{C}) \cong \mathbb{C} \oplus \mathcal{E}$. This follows from the following lemma.

Lemma 5.8. Let $Q$ be the quadric given by the equation $\sum_{i=1}^{k} a_{i}^{2}=0$ in $\mathbb{P}^{n-1}$ with coordinates $\left[a_{1}, \ldots, a_{n}\right]$ and consider the automorphism $\gamma$ of $\mathbb{P}^{n-1}$ given by $\gamma\left[a_{1}, a_{2}, a_{3}, \ldots, a_{n}\right]=$ $\left[a_{2}, a_{1}, a_{3}, \ldots, a_{n}\right]$. If $k$ is odd, then $\gamma$ acts trivially on $H^{*}(Q, \mathbb{C})$. If $k$ is even, $\gamma$ acts trivially on $H^{2 j}(Q)$ for $j \neq 2 n-k-2$ and the action on the two dimensional space $H^{2 n-k-2}(Q, \mathbb{C})$ has eigenvalues 1 and -1 .

Proof. The quadric $Q$ is the join of the nonsingular quadric $\tilde{Q}$ in $\mathbb{P}^{k-1} \subset \mathbb{P}^{n-1}$ given by $\sum_{i=1}^{k} a_{i}^{2}=0$ and the linear subspace $L$ of dimension $n-k-1$ given by $a_{1}=\cdots=a_{k}=0$. Now $Q-L$ is an affine space bundle over $\tilde{Q}$ of fiber dimension $n-k$. Thus, $H_{c}^{i}(Q-L, \mathbb{C})=$ $H^{i-2 n+2 k}(\tilde{Q}, \mathbb{C})$. As $\tilde{Q}$ and $L$ only have (compactly supported) cohomology in even degrees we conclude that

$$
H^{i}(Q, \mathbb{C})=H^{i-2 n+2 k}(\tilde{Q}, \mathbb{C}) \oplus H^{i}(L, \mathbb{C})
$$

The automorphism $\gamma$ of course acts trivially on the cohomology of $\mathbb{P}^{n-1}$ and hence it acts trivially on the cohomology of $L$ (it even acts trivially on $L$ itself). Thus we are reduced to consider the action of $\gamma$ on the cohomology of $\tilde{Q}$. The action on the non-primitive cohomology is trivial and so the only possibly nontrivial action is on $H_{\text {prim }}^{k-2}(\tilde{Q}, \mathbb{C})$. If $k$ is odd, this group is zero and so we are reduced to the case of $k$ even.

Assume that $k$ is even and $k=2 k_{0}$. The variety of $\left(k_{0}-1\right)$-planes contained in $\tilde{Q}$ has two disjoint irreducible components, which can be identified with the two disjoint irreducible components of the variety of maximal isotropic spaces in $\mathbb{C}^{2 k_{0}}$ (equipped with the standard bilinear form). It is clear that $\gamma$, regarded as an element in $O_{2 k_{0}}-S O_{2 k_{0}}$, interchanges these two irreducible components. Now Reid in [Re, Theorem 1.12] has shown that $H^{k-2}(\tilde{Q}, \mathbb{C})=$ $\operatorname{span}(a, b)$, where $a$ and $b$ are the classes of $\left(k_{0}-1\right)$-planes from the two families respectively. Thus our lemma follows.

The fiber $\widetilde{\tau}_{i}^{-1}\left(x^{\prime}\right)$ is a quadric bundle over $\tau_{i}^{-1}\left(x^{\prime}\right) \cong \operatorname{SpGr}(i-j, 2 n-2 j)$ with fibers a quadric $\sum_{s=1}^{j} b_{s}^{2}=0$ in $\mathbb{P}^{i-1}$. The action of $A^{\prime}\left(x^{\prime}\right)$ on $H^{*}\left(\widetilde{\tau}_{i}^{-1}\left(x^{\prime}\right), \mathbb{C}\right)$ is entirely similar. One checks readily that (5.6) and (5.7) hold. 
5.3. Proof of Theorem 5.1, Theorem 5.2 and Proposition 5.3. The proofs are based on some well-known principle by making use of the resolutions $\sigma_{i}, \tau_{i}$ of $\overline{\mathcal{O}}_{2^{i} 1^{2 n-2 i+1}}, \overline{\mathcal{O}}_{2^{i} 1^{2 n-2 i}}$ defined in $\$ 5.1$, and the maps $\tilde{\sigma}_{i}, \tilde{\tau}_{i}$ introduced in $\$ 5.2$ for $i$ even. We give the detailed proof for Theorem 5.1 in the following. The proofs of Theorem 5.2 and Proposition 5.3 are entirely similar and we leave that to the readers. We mention only that the proof of Proposition 5.3 indicates the following

$$
\left(t_{l, k}^{i}\right)_{n}=\left(t_{l-j, k}^{i-j}\right)_{n-j} \text { for } j \leq l<i .
$$

Here the numbers are written as $\left(t_{l, k}^{i}\right)_{n}$ to indicate that the ambient symmetric pair is $(S L(2 n+1), S O(2 n+1))$ and they are defined in (5.3) $)$.

To prove Theorem 5.1, we begin with the following lemma.

Lemma 5.9. Let $i \in[1, n]$. Assume that $t_{j k}^{i^{\prime}}=\left(t_{j k}^{i^{\prime}}\right)^{\prime}$ for all $j, k$, and all $i^{\prime} \leq i$ in (5.3) and (5.4). Then we have

$$
\mathcal{H}_{x}^{l} \mathrm{IC}\left(\mathcal{O}_{2^{i} 1^{2 n-2 i+1}}, \mathbb{C}\right) \simeq \mathcal{H}_{x^{\prime}}^{l} \mathrm{IC}\left(\mathcal{O}_{2^{i} 1^{2 n-2 i}}^{\prime}, \mathbb{C}\right)
$$

for $x \in \mathcal{O}_{2^{j} 1^{2 n-2 j+1}}, x^{\prime} \in \mathcal{O}_{2^{j} 1^{2 n-2 j}}^{\prime}$ and $j \leq i$.

Proof. We prove the lemma by induction on $i$. The case when $i=1$ is clear. In fact, we only need to check the conclusion of the lemma for $j=0$. We have

$$
\begin{aligned}
& \left(\sigma_{1}\right)_{*} \mathbb{C}[-]=\operatorname{IC}\left(\mathcal{O}_{2^{1} 1^{2 n-1}}, \mathbb{C}\right) \oplus \bigoplus_{k \geq 0} \mathrm{IC}\left(\mathcal{O}_{1^{2 n+1}}, \mathbb{C}^{t_{0 k}^{1}}\right)[ \pm k] . \\
& \left.\left(\tau_{1}\right)_{*} \mathbb{C}[-]=\mathrm{IC}\left(\mathcal{O}_{2^{1} 1^{2 n-2}}^{\prime}, \mathbb{C}\right) \oplus \bigoplus_{k \geq 0} \mathrm{IC}\left(\mathcal{O}_{1^{2 n}}, \mathbb{C}^{\left(t_{0 k}^{1}\right.}\right)^{\prime}\right)[ \pm k] .
\end{aligned}
$$

It is clear that $\mathcal{H}_{0}^{l} \mathrm{IC}\left(\mathcal{O}_{2^{1} 1^{2 n-1}}, \mathbb{C}\right) \cong \mathcal{H}_{0}^{l} \mathrm{IC}\left(\mathcal{O}_{2^{1} 1^{2 n-2}}^{\prime}, \mathbb{C}\right)$ as they are determined by the cohomology of $\sigma_{1}^{-1}(0) \cong \tau_{1}^{-1}(0)$ and the numbers $\left(t_{0 k}^{1}\right)=\left(t_{0 k}^{1}\right)^{\prime}$ in the same way.

By induction hypothesis, we can assume that for $s<i$

$$
\mathcal{H}_{x}^{k} \mathrm{IC}\left(\mathcal{O}_{2^{s} 1^{2 n-2 s+1}}, \mathbb{C}\right) \simeq \mathcal{H}_{x^{\prime}}^{k} \mathrm{IC}\left(\mathcal{O}_{2^{s} 1^{2 n-2 s}}^{\prime}, \mathbb{C}\right)
$$

Recall that

$$
\left(\sigma_{i}\right)_{*} \mathbb{C}[-]=\mathrm{IC}\left(\mathcal{O}_{2^{i} 1^{2 n-2 i+1}}, \mathbb{C}\right) \oplus \bigoplus_{s<i} \mathrm{IC}\left(\mathcal{O}_{2^{s} 1^{2 n-2 s+1}}, \mathbb{C}^{t_{s k}^{i}}\right)[ \pm k] .
$$

This implies that the stalks of $\mathrm{IC}\left(\mathcal{O}_{2^{i} 1^{2 n-2 i+1}}, \mathbb{C}\right)$ are uniquely determined by the stalks of $\mathrm{IC}\left(\mathcal{O}_{2^{s} 1^{2 n-2 s+1}}, \mathbb{C}\right)$ for $s<i$, the cohomology groups of the fibers of the map $\sigma_{i}$, and the numbers $t_{s k}^{i}, s<i$. Similarly, the stalks of $\operatorname{IC}\left(\mathcal{O}_{2^{i} 1^{2 n-2 i}}^{\prime}, \mathbb{C}\right)$ are uniquely determined, in the same way, by the stalks of $\mathrm{IC}\left(\mathcal{O}_{2^{s} 1^{2 n-2 s}}^{\prime}, \mathbb{C}\right)$ for $s<i$, the cohomology groups of the fibers of the map $\tau_{i}$, and the numbers $\left(t_{s k}^{i}\right)^{\prime}, s<i$. Now the desired claim follows form (5.11), (5.1), and the assumption that $t_{j k}^{i}=\left(t_{j k}^{i}\right)^{\prime}$.

By the lemma above, to prove Theorem 5.1, it suffices to show the following

Lemma 5.10. We have $t_{j k}^{i}=\left(t_{j k}^{i}\right)^{\prime}$. 
Proof. We argue by induction on $i$. The case when $i=1$ is easy to check, i.e. we have that $t_{0, k}^{1}=\left(t_{0, k}^{1}\right)^{\prime}=1$ when $k \in\left[0, s_{1 j}\right]$ and $k$ is even, and $t_{0 k}^{1}=\left(t_{0 k}^{1}\right)^{\prime}=0$ otherwise.

So by induction hypothesis we can assume that for all $s<i, t_{j k}^{s}=\left(t_{j k}^{s}\right)^{\prime}$. By lemma [5.9, we have for $s<i$,

$$
\mathcal{H}_{x}^{k} \mathrm{IC}\left(\mathcal{O}_{2^{s} 1^{2 n-2 s+1}}, \mathbb{C}\right) \simeq \mathcal{H}_{x^{\prime}}^{k} \mathrm{IC}\left(\mathcal{O}_{2^{s} 1^{2 n-2 s}}^{\prime}, \mathbb{C}\right)
$$

for $x \in \mathcal{O}_{2^{j} 1^{2 n-2 j+1}}, x^{\prime} \in \mathcal{O}_{2^{j} 1^{2 n-2 j}}^{\prime}$ and $j \leq s$.

We show that $t_{j k}^{i}=\left(t_{j k}^{i}\right)^{\prime}$ by induction on $j$. The case $j=i$ is clear. So by induction, we can assume that $t_{j^{\prime} k}^{i}=\left(t_{j^{\prime} k}^{i}\right)^{\prime}$ holds for $j<j^{\prime} \leq i$. Then, for $x_{j} \in \mathcal{O}_{2^{j} 1^{2 n-2 j+1}}$, we have

$$
\left.\left(\sigma_{i}\right)_{*} \mathbb{C}[-]\right|_{x_{j}}=\left.\left.\mathrm{IC}\left(\mathcal{O}_{2^{i} 1^{2 n-2 i+1}}, \mathbb{C}\right)\right|_{x_{j}} \oplus \bigoplus_{j \leq j^{\prime}<i} \operatorname{IC}\left(\mathcal{O}_{2^{j^{\prime}} 1^{2 n-2 j^{\prime}+1}}, \mathbb{C}^{t_{j^{\prime} k}^{i}}\right)[ \pm k]\right|_{x_{j}}
$$

Since the stalk $\left.\mathrm{IC}\left(\mathcal{O}_{2^{i} 1^{2 n-2 i+1}}, \mathbb{C}\right)\right|_{x_{j}}$ is concentrated in degree $<-\operatorname{dim} \mathcal{O}_{2^{j} 1^{2 n-2 j+1}}$ and $\mathrm{IC}\left(\mathcal{O}_{2^{j} 1^{2 n-2 j+1}}, \mathbb{C}^{t_{j k}^{i}}\right)[-k]$ is concentrated in degree $\geq-\operatorname{dim} \mathcal{O}_{2^{j} 1^{2 n-2 j+1}}$, the decomposition in (5.13) implies the multiplicity numbers $t_{j k}^{i}$ are uniquely determined by the cohomology of $\sigma_{i}^{-1}\left(x_{j}\right)$ and the stalks $\left.\operatorname{IC}\left(\mathcal{O}_{2^{j^{\prime}}{ }^{2 n-2 j^{\prime}+1}}, \mathbb{C}^{t_{j^{\prime} k}^{i}}\right)\right|_{x_{j}}$ for $j<j^{\prime}<i$. The numbers $\left(t_{j k}^{i}\right)^{\prime}$ are determined, in the same manner, by the corresponding data. Now since $t_{j^{\prime} k}^{i}=\left(t_{j^{\prime} k}^{i}\right)^{\prime}$ for $j^{\prime}>j$ (by induction) the desired equality $t_{j k}^{i}=\left(t_{j k}^{i}\right)^{\prime}$ follows from (5.12).

\section{Cohomology of Fano varieties}

In this section we compute the cohomology of Fano varieties of $k$-planes in the smooth complete intersection of two quadrics in $\mathbb{P}^{2 n}$. We denote these Fano varieties by $\operatorname{Fano}_{k}^{2 n}$. Note that Fano $_{0}^{2 n}$ is the smooth complete intersection of two quadrics in $\mathbb{P}^{2 n}$.

Let $\operatorname{Gr}(k, n)$ denote the Grassmannian variety of $k$-dimensional subspaces in $\mathbb{C}^{n}$. Let

$$
g_{k, n}(q):=\sum \operatorname{dim} H^{2 l}(\operatorname{Gr}(k, n), \mathbb{C}) q^{l}=\frac{\prod_{l=n-k+1}^{n}\left(1-q^{l}\right)}{\prod_{l=1}^{k}\left(1-q^{l}\right)}
$$

be the Poincare polynomial of $\operatorname{Gr}(k, n)$.

Recall the monodromy representations $L_{i}$ which were defined in $\$ 2.3$, The cohomology of the Fano variety $\mathrm{Fano}_{i-1}^{2 n}$ is described as follows.

Theorem 6.1. We have

$$
\begin{gathered}
H^{2 k+1}\left(\mathrm{Fano}_{i-1}^{2 n}, \mathbb{C}\right)=0, \\
H^{2 k}\left(\mathrm{Fano}_{i-1}^{2 n}, \mathbb{C}\right) \cong \bigoplus_{j=0}^{i} L_{j}^{\oplus M_{i}(k, j)},
\end{gathered}
$$

where $M_{i}(k, j)$ is the coefficient of $q^{k-j(n-i)}$ in $g_{i-j, 2 n-i-j}(q)$. 
6.1. Fano varieties and resolutions for $\overline{\mathcal{O}}_{2^{i} 1^{2 n-2 i+1}}$. We start with the following simple observation, which is a direct consequence of Theorem 2.2 .

Let $\pi: \Sigma \rightarrow \mathfrak{g}_{1}^{r s}$ be a family of smooth projective varieties over $\mathfrak{g}_{1}^{r s}$ and let $R^{k} \pi_{*} \mathbb{C}$ be the corresponding local system on $\mathfrak{g}_{1}^{r s}$. Suppose that the Fourier transform of $\operatorname{IC}\left(\mathfrak{g}_{1}, R^{k} \pi_{*} \mathbb{C}\right)$ is supported on $\overline{\mathcal{O}}_{2^{n} 1}$ and is given by

$$
\mathfrak{F}\left(\mathrm{IC}\left(\mathfrak{g}_{1}, R^{k} \pi_{*} \mathbb{C}\right)\right)=\bigoplus_{j=0}^{n} \mathrm{IC}\left(\mathcal{O}_{2^{j} 1^{2 n-2 j+1}}, \mathbb{C}^{m_{k j}}\right) .
$$

Then the cohomology of the fiber $\Sigma_{x}:=\pi^{-1}(x)$ over $x \in \mathfrak{g}_{1}^{r s}$ satisfies

$$
H^{k}\left(\Sigma_{x}, \mathbb{C}\right) \cong \bigoplus_{j=0}^{n} L_{j}^{\oplus m_{k j}}
$$

Moreover, the isomorphism above is compatible with the monodromy actions.

Let us apply this observation to the following situation. Consider the maps

$$
\check{\sigma}_{i}:\left\{\left(x, 0 \subset V_{i} \subset V_{i}^{\perp} \subset \mathbb{C}^{2 n+1}\right) \mid x \in \mathfrak{g}_{1}, x V_{i} \subset V_{i}^{\perp}\right\} \rightarrow \mathfrak{g}_{1} .
$$

Note that for $x \in \mathfrak{g}_{1}^{r s}$, we have $\check{\sigma}_{i}^{-1}(x) \cong \operatorname{Fano}_{i-1}^{2 n}$, the Fano variety of $(i-1)$-planes in the smooth complete intersection of two quadrics $Q(v)=0$ and $\langle x v, v\rangle_{Q}=0$ in $\mathbb{P}^{2 n}$.

Let us consider $\pi_{i}=\left.\check{\sigma}_{i}\right|_{\check{\sigma}_{i}^{-1}\left(\mathfrak{g}_{1}^{r s}\right)}$, which is a smooth family of Fano varieties, and consider the corresponding local system $R^{k} \pi_{i *} \mathbb{C}$. Recall that we have Reeder's resolutions $\sigma_{i}$ for $\overline{\mathcal{O}}_{2^{i} 1^{2 n-2 i+1}}($ see $\$ 5.1$ ) and

$$
\sigma_{i *} \mathbb{C}[i(2 n+1-i)]=\bigoplus_{j=0}^{i} \bigoplus_{k=0}^{2(i-j)(n-i)} \operatorname{IC}\left(\mathcal{O}_{2^{j}} 1^{2 n-2 j+1}, \mathbb{C}^{t_{j k}^{i}}\right)[ \pm k] .
$$

Since the Fourier transform of $\operatorname{IC}\left(\mathcal{O}_{2^{j} 1^{2 n-2 j+1}}, \mathbb{C}\right)$ is supported on all of $\mathfrak{g}_{1}$ for all $j$ (Theorem 2.2), the equation

$$
\mathfrak{F}\left(\check{\sigma}_{i *} \mathbb{C}[-]\right) \cong \sigma_{i *} \mathbb{C}[-]
$$

implies that

$$
\begin{aligned}
& \mathfrak{F}\left(\check{\sigma}_{i *} \mathbb{C}[-]\right) \cong \bigoplus_{k=0}^{4 i(n-i)} \mathfrak{F}\left(\mathrm{IC}\left(\mathfrak{g}_{1}, R^{k} \pi_{i *} \mathbb{C}\right)[-k+2 i(n-i)]\right) \\
& \cong \bigoplus_{j=0}^{i} \bigoplus_{k=0}^{2(i-j)(n-i)} \mathrm{IC}\left(\mathcal{O}_{2^{j} 1^{2 n-2 j+1}}, \mathbb{C}^{t_{j k}^{i}}\right)[ \pm k]
\end{aligned}
$$

here $2 i(n-i)=\operatorname{dim} \check{\sigma}_{i}^{-1}(x)=\operatorname{dim} \operatorname{Fano}_{i-1}^{2 n}$, for $x \in \mathfrak{g}_{1}^{r s}$.

Hence we see that $\mathfrak{F}\left(\mathrm{IC}\left(\mathfrak{g}_{1}, R^{k} \pi_{i *} \mathbb{C}\right)\right)$ is supported on $\overline{\mathcal{O}}_{2^{i} 1^{2 n-2 i+1}}$, and has the form

$$
\mathfrak{F}\left(\mathrm{IC}\left(\mathfrak{g}_{1}, R^{k} \pi_{i *} \mathbb{C}\right)\right)=\bigoplus_{j=0}^{i} \mathrm{IC}\left(\mathcal{O}_{2^{j} 1^{2 n-2 j+1}}, \mathbb{C}^{t_{j,|2 i(n-i)-k|}^{i}}\right) .
$$


So by the observation above and Theorem 2.2, we deduce that the cohomology of the Fano varieties $\operatorname{Fano}_{i-1}^{2 n}$ is given by

$$
H^{k}\left(\mathrm{Fano}_{i-1}^{2 n}, \mathbb{C}\right) \cong \bigoplus_{j=0}^{i} L_{j}^{\oplus t_{j,|2 i(n-i)-k|}^{i}}
$$

6.2. The numbers $t_{j k}^{i}$. In this subsection let us again use the notation $\left(t_{j k}^{i}\right)_{n}$ for the numbers $\left(t_{j k}^{i}\right)$ in (6.3) to indicate that the ambient symmetric pair is $(S L(2 n+1), S O(2 n+1))$.

Lemma 6.2. We have $\left(t_{j k}^{i}\right)_{n}=0$ for odd $k$.

Proof. In the decomposition (6.3), we take the stalk $\mathcal{H}_{x_{j}}^{l}$ on both sides for odd $l$, where $x_{j} \in$ $\mathcal{O}_{2^{j} 1^{2 n+1-2 j}}$. Since $i(2 n-i+1)$ is even and $H^{\text {odd }}\left(\sigma_{i}^{-1}\left(x_{j}\right), \mathbb{C}\right)=0$, we have that $\mathcal{H}_{x_{j}}^{l} \sigma_{i *} \mathbb{C}[i(2 n+$ $1-i)]=0$ for all odd $l$. Suppose that there exists $k$ odd such that $t_{j k}^{i} \neq 0$, then there exists an odd $k$ such that $\mathcal{H}_{x_{j}}^{l} \mathrm{IC}\left(\mathcal{O}_{2^{j} 1^{2 n-2 j+1}}, \mathbb{C}^{t_{j k}^{i}}\right)[ \pm k] \neq 0$ (note that $\mathcal{H}_{x_{j}}^{-j(2 n-j+1)} \mathrm{IC}\left(\mathcal{O}_{2^{j} 1^{2 n-2 j+1}}, \mathbb{C}\right) \neq$ $0)$. This is a contradiction. Thus the lemma is proved.

Recall from (5.10) that we have $\left(t_{l, k}^{i}\right)_{n}=\left(t_{l-j, k}^{i-j}\right)_{n-j}$ for $j \leq l<i$. This implies, in particular, that $\left(t_{j k}^{i}\right)_{n}=\left(t_{0, k}^{i-j}\right)_{n-j}$ for $j \geq 1$. Since $\left(t_{i, k}^{i}\right)_{n}=\delta_{0, k}$, the determination of $\left(t_{j k}^{i}\right)_{n}$ are reduced to that of $\left(t_{0, k}^{i}\right)_{n}$. In the following we describe how to determine the latter numbers inductively. The dimensions of the stalks $\mathcal{H}_{0}^{k} \mathrm{IC}\left(\mathcal{O}_{2^{i} 1^{2 n+1-2 i}}, \mathbb{C}\right)$ can be determined simultaneously (in 6.3 we determine these dimensions directly, see (6.8))

Recall that $\mathcal{H}_{0}^{\text {odd }} \mathrm{IC}\left(\mathcal{O}_{2^{j} 1^{2 n-2 j+1}}, \mathbb{C}\right)=0$ (see Corollary 4.1). Note also that $\mathcal{H}_{0}^{2 k} \mathrm{IC}\left(\mathcal{O}_{2^{j} 1^{2 n-2 j+1}}, \mathbb{C}\right) \neq 0$ implies that $-\operatorname{dim} \mathcal{O}_{2^{j} 1^{2 n-2 j+1}} \leq 2 k \leq-1$. Let us write

$$
\begin{gathered}
m_{j}=\left(\operatorname{dim} \mathcal{O}_{2^{j} 1^{2 n-2 j+1}}\right) / 2=j(2 n-j+1) / 2, \\
f_{j}(q)=\sum_{k=-m_{j}}^{-1}\left(\operatorname{dim} \mathcal{H}_{0}^{2 k} \mathrm{IC}\left(\mathcal{O}_{2^{j} 1^{2 n-2 j+1}}, \mathbb{C}\right)\right) q^{k}, \\
\text { and } o_{j, 2 n+1}(q)=\sum_{k=0}^{d_{j}} \operatorname{dim} H^{2 k}(\operatorname{OGr}(j, 2 n+1), \mathbb{C}) q^{k},
\end{gathered}
$$

where $d_{j}=\operatorname{dim} \operatorname{OGr}(j, 2 n+1)=j(4 n-3 j+1) / 2$. The polynomials $o g_{j, 2 n+1}(q)$ are known, i.e.

$$
\operatorname{og}_{i, 2 n+1}(q)=\frac{\left(1-q^{2(n-i+1)}\right)\left(1-q^{2(n-i+2)}\right) \cdots\left(1-q^{2 n}\right)}{(1-q)\left(1-q^{2}\right) \cdots\left(1-q^{i}\right)}
$$

Note that $\sigma_{i}^{-1}(0) \cong \operatorname{OGr}(i, 2 n+1)$. In view of Lemma 6.2, the decomposition (6.3) implies that

$$
o g_{i, 2 n+1}(q) q^{-i(2 n-i+1) / 2}=f_{i}(q)+\sum_{j=1}^{i-1} f_{j}(q) \sum_{k=0}^{(i-j)(n-i)}\left(t_{j, 2 k}^{i}\right)_{n} q^{ \pm k}+\sum_{k=0}^{i(n-i)}\left(t_{0,2 k}^{i}\right)_{n} q^{ \pm k}
$$

Note that $f_{0}(q)=1$. It is easy to check using (6.5) that

$$
\left(t_{0,2 k}^{1}\right)_{n}=1 \text { for } 0 \leq k \leq n-1,\left(t_{0,2 k}^{1}\right)_{n}=0 \text { otherwise, and } f_{1}(q)=q^{-n} \text {. }
$$


This completes the determination of the numbers $\left(t_{j k}^{i}\right)_{n}$ and $f_{i}(q)^{\prime}$ 's for $n=1$, and for all $n$ and $i=1$. By induction on $n$, we can assume that the numbers $\left(t_{j k}^{i}\right)_{n^{\prime}}$ have been determined for all $n^{\prime}<n$. This implies that $\left(t_{j k}^{i}\right)_{n}$ for $j \geq 1$ have been determined. We determine now the numbers $\left(t_{0 k}^{i}\right)_{n}$ by induction on $i$. We can assume that all $f_{i^{\prime}}(q), i^{\prime}<i$, has been determined. Note that $f_{i}(q)$ is concentrated in negative degrees. Thus we can determine the numbers $\left(t_{0,2 k}^{i}\right)_{n}$ from (6.5) and then determine $f_{i}(q)$. We have shown that

$$
\text { the equations (6.5) determine } f_{i}(q) \text { 's and }\left(\sum_{k=0}^{i(n-i)}\left(t_{0,2 k}^{i}\right)_{n} q^{ \pm k}\right) \text { 's uniquely. }
$$

6.3. The functions $f_{i}(q)$. In fact, the functions $f_{i}(q)$ can be determined directly making use of our identification of $\mathcal{H}_{0}^{k} \mathrm{IC}\left(\mathcal{O}_{2^{i} 1^{2 n+1-2 i}}, \mathbb{C}\right)$ with $\mathcal{H}_{0}^{k} \mathrm{IC}\left(\mathcal{O}_{2^{i} 1^{2 n-2 i}}^{\prime}, \mathbb{C}\right)$ (see Theorem 5.1), where $\mathcal{O}_{2^{i} 1^{2 n-2 i}}^{\prime} \subset \mathcal{N}_{\mathfrak{s p}(2 n)}$, and the classical Springer correspondence.

Lemma 6.3. We have that

$$
f_{i}(q)=q^{-i(2 n-i+1) / 2} g_{[i / 2], n}\left(q^{2}\right),
$$

where $[i / 2]$ is the integer part of $i / 2$. Namely

$$
\begin{gathered}
\operatorname{dim} \mathcal{H}_{0}^{k-i(2 n-i+1)} \operatorname{IC}\left(\mathcal{O}_{2^{i}} 1^{2 n-2 i+1}, \mathbb{C}\right)=\operatorname{dim} H^{k / 2}\left(\operatorname{Gr}\left(\left[\frac{i}{2}\right], n\right), \mathbb{C}\right) \text { if } k \equiv 0(\bmod 4), \\
\operatorname{dim} \mathcal{H}_{0}^{k-i(2 n-i+1)} \operatorname{IC}\left(\mathcal{O}_{2^{i}} 1^{2 n-2 i+1}, \mathbb{C}\right)=0 \text { otherwise. }
\end{gathered}
$$

Proof. We have

$$
\operatorname{dim} \mathcal{H}_{0}^{k} \mathrm{IC}\left(\mathcal{O}_{2^{i} 1^{2 n-2 i+1}}, \mathbb{C}\right)=\operatorname{dim} \mathcal{H}_{0}^{k} \operatorname{IC}\left(\mathcal{O}_{2^{i} 1^{2 n-2 i}}^{\prime}, \mathbb{C}\right) \stackrel{4.233}{=}\left[V_{\left(\mathcal{O}_{2^{i} 12 n-2 i}^{\prime}, \mathbb{C}\right)}: H^{k+2 n^{2}}(\mathcal{B}, \mathbb{C})\right],
$$

where $V_{\left(\mathcal{O}_{2 i_{12 n-2 i}}^{\prime}, \mathbb{C}\right)}$ is the representation of $W_{n}$ attached to the pair $\left(\mathcal{O}_{2^{i} 1^{2 n-2 i}}^{\prime}, \mathbb{C}\right)$ under Springer correspondence (see $\$ 4.3)$. The numbers $\left[V_{\left(\mathcal{O}_{2 i 12 n-2 i}^{\prime}, \mathbb{C}\right)}: H^{k+2 n^{2}}(\mathcal{B}, \mathbb{C})\right]$ are the socalled fake degrees and they have been computed explicitly by Lusztig in [L2]. In fact, let us write

Using (4.25) and [L2], we see that

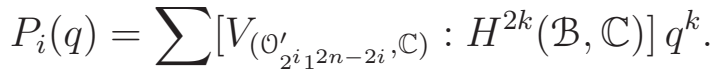

$$
P_{i}(q)=q^{n^{2}-n i+i(i-1) / 2} g_{[i / 2], n}\left(q^{2}\right) .
$$

Now $\operatorname{dim} \mathcal{H}_{0}^{k-i(2 n-i+1)} \mathrm{IC}\left(\mathcal{O}_{2^{i} 1^{2 n-2 i+1}}, \mathbb{C}\right)$ is the coefficient of $q^{\frac{k-i(2 n-i+1)+2 n^{2}}{2}}$ in $P_{i}(q)$, which is the coefficient of $q^{k / 2}$ in $g_{[i / 2], n}\left(q^{2}\right)$. The equation (6.8) follows.

6.4. Proof of Theorem 6.1. The equation (6.1) in the theorem follows from (6.4) and Lemma 6.2. The equation (6.2) in the theorem follows from (6.4) and the following statement about the numbers $t_{j, 2 k}^{i}$.

Proposition 6.4. We have

$$
\sum_{k=0}^{(i-j)(n-i)}\left(t_{j, 2 k}^{i}\right)_{n} q^{ \pm k}=q^{-(i-j)(n-i)} g_{i-j, 2 n-i-j}(q) .
$$


In view of (6.6), the proposition above follows from (6.5), (6.7), and the following equation

$$
o g_{i, 2 n+1}(q)=\sum_{j=0}^{i} q^{(i-j)(i-j+1) / 2} g_{[j / 2], n}\left(q^{2}\right) g_{i-j, 2 n-i-j}(q), \text { where } g_{0, n}(q)=1 \text {. }
$$

Proof of (6.9). The proof given here was kindly supplied to us by Dennis Stanton.

Define $(A ; q)_{l}:=\prod_{k=0}^{l-1}\left(1-A q^{k}\right)$ and $\left[\begin{array}{c}n \\ j\end{array}\right]_{q}:=\frac{(q ; q)_{n}}{(q ; q)_{j}(q ; q)_{n-j}}$. Let us write

$$
S=\sum_{j=0}^{i} q^{(i-j)(i-j+1) / 2} g_{[j / 2], n}\left(q^{2}\right) g_{i-j, 2 n-i-j}(q)
$$

We have

$$
\begin{gathered}
\left.S=\sum_{j=0}^{[i / 2]} q^{(i-2 j-1)(i-2 j) / 2}\left[\begin{array}{c}
n \\
j
\end{array}\right]_{q^{2}}\left(\begin{array}{c}
2 n-i-2 j-1 \\
i-2 j-1
\end{array}\right]_{q}+q^{i-2 j}\left[\begin{array}{c}
2 n-i-2 j \\
i-2 j
\end{array}\right]_{q}\right) \\
=\frac{q^{\left(\begin{array}{c}
i \\
2
\end{array}\right)(q ; q)_{2 n-i-1}}}{(q ; q)_{2 n-2 i}(q ; q)_{i}} \sum_{j=0}^{[i / 2]}(-1)^{j} \frac{\left(q^{-2 n} ; q^{2}\right)_{j}\left(q^{-i} ; q\right)_{j}\left(q^{1-i} ; q\right)_{j} q^{j(j+2 i-2 n+4)}}{\left(q^{2} ; q^{2}\right)_{j}\left(q^{-2 n+i+1} ; q^{2}\right)_{j}\left(q^{-2 n+i+2} ; q^{2}\right)_{j}}\left(1-q^{2 n-4 j}\right) .
\end{gathered}
$$

Consider the terminating very well-poised basic hypergeometric series which, by definition, is given by

$$
\begin{aligned}
& { }_{6} \phi_{5}\left(\begin{array}{c}
a, q^{2} a^{1 / 2},-q^{2} a^{1 / 2}, b, c, q^{-2 m} \\
a^{1 / 2},-a^{1 / 2}, a q^{2} / b, a q^{2} / c, a q^{2 m+2}
\end{array} ; q^{2}, \frac{a q^{2 m+2}}{b c}\right) \\
& =\sum_{j=0}^{\infty} \frac{\left(a ; q^{2}\right)_{j}\left(q^{2} a^{1 / 2} ; q^{2}\right)_{j}\left(-q^{2} a^{1 / 2} ; q^{2}\right)_{j}\left(b ; q^{2}\right)_{j}\left(c ; q^{2}\right)_{j}\left(q^{-2 m} ; q^{2}\right)_{j}}{\left(q^{2} ; q^{2}\right)_{j}\left(a^{1 / 2} ; q^{2}\right)_{j}\left(-a^{1 / 2} ; q^{2}\right)_{j}\left(a q^{2} / b ; q^{2}\right)_{j}\left(a q^{2} / c ; q^{2}\right)_{j}\left(a q^{2 m+2} ; q^{2}\right)_{j}}\left(\frac{a q^{2 m+2}}{b c}\right)^{j} .
\end{aligned}
$$

Note that if $m>0$, then $\left(q^{-2 m} ; q^{2}\right)_{j}=0$ for $j>m$ and thus the sum above is finite. Take

$$
a=q^{-2 n} ; m=i / 2 \text { and } b=q^{1-i} \text { for even } i \text {; and } m=(i-1) / 2, b=q^{-i} \text { for odd } i \text {. }
$$

One checks that

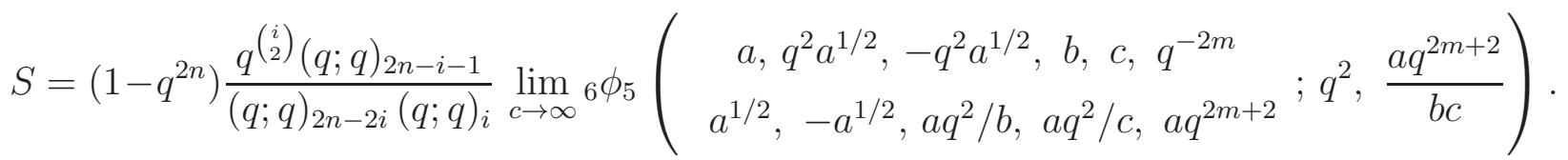

Now use the summation formula (see [GR, Appendix (II.21)])

$$
{ }_{6} \phi_{5}\left(\begin{array}{c}
a, q^{2} a^{1 / 2},-q^{2} a^{1 / 2}, b, c, q^{-2 m} \\
a^{1 / 2},-a^{1 / 2}, a q^{2} / b, a q^{2} / c, a q^{2 m+2}
\end{array} ; q^{2}, \frac{a q^{2 m+2}}{b c}\right)=\frac{\left(a q^{2} ; q^{2}\right)_{m}\left(a q^{2} / b c ; q^{2}\right)_{m}}{\left(a q^{2} / b ; q^{2}\right)_{m}\left(a q^{2} / c ; q^{2}\right)_{m}}
$$

we get

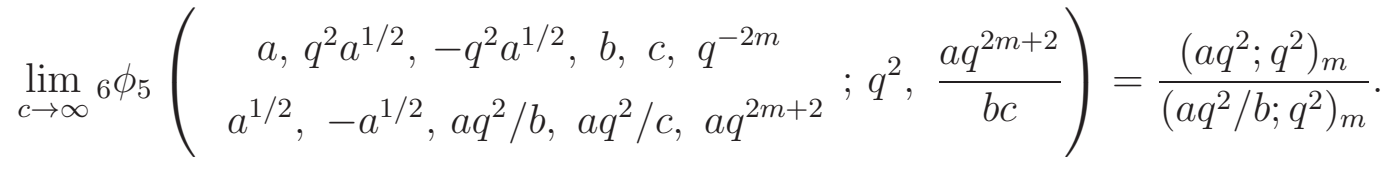


Thus

$$
S=\left(1-q^{2 n}\right) \frac{q^{\left(\begin{array}{c}
i \\
2
\end{array}\right)}(q ; q)_{2 n-i-1}\left(a q^{2} ; q^{2}\right)_{m}}{(q ; q)_{2 n-2 i}(q ; q)_{i}\left(a q^{2} / b ; q^{2}\right)_{m}}=o g_{i, 2 n+1}(q)
$$

Example 6.5 (Cohomology of $\mathrm{Fano}_{1}^{2 n}$ ). The cohomology of $\mathrm{Fano}_{1}^{2 n}$, the Fano variety of lines in the smooth complete intersection of two quadrics in $\mathbb{P}^{2 n}$, can be described as follows:

$$
\begin{gathered}
H^{2 k+1}\left(\mathrm{Fano}_{1}^{2 n}, \mathbb{C}\right)=0 \\
H^{2(4 n-8-k)}\left(\mathrm{Fano}_{1}^{2 n}, \mathbb{C}\right)=H^{2 k}\left(\mathrm{Fano}_{1}^{2 n}, \mathbb{C}\right)= \begin{cases}\mathbb{C}^{\left[\frac{k+2}{2}\right]} & \text { if } 0 \leq k \leq n-3 \\
\mathbb{C}^{\left[\frac{k+2}{2}\right]} \oplus L_{1} & \text { if } n-2 \leq k \leq 2 n-5 \\
\mathbb{C}^{\left[\frac{k+2}{2}\right]} \oplus L_{1} \oplus L_{2} & \text { if } k=2 n-4 .\end{cases}
\end{gathered}
$$

\section{REFERENCES}

[A] N. A'Campo, Tresses, monodromie et le groupe symplectique, Comment. Math. Helv. 54 (1979), no. $2,318-327$.

[BG] M. Bhargava and B. H. Gross, The average size of the 2-Selmer group of Jacobians of hyperelliptic curves having a rational Weierstrass point, in: Automorphic Representations and L-Functions, pp. 23-91, Tata Inst. Fundam. Res. Stud. Math. 22, Tata Inst. Fund. Res., Mumbai, (2013).

[C] C. Casagrande, Rank 2 quasiparabolic vector bundles on $\mathbb{P}^{1}$ and the variety of linear subspaces contained in two odd-dimensional quadrics, Mathematische Zeitschrift 280 (2015), no. 3-4, 981988.

[CVX1] T. H. Chen, K. Vilonen, and T. Xue, Hessenberg varieties, intersections of quadrics, and the Springer correspondence, Trans. Amer. Math. Soc. 373 (2020), no. 4, 2427-2461.

[CVX2] T. H. Chen, K. Vilonen, and T. Xue, On the cohomology of Fano varieties and the Springer correspondence (with an appendix by Dennis Stanton), Adv. in Math. 318 (2017), 515-533.

[CVX3] T. H. Chen, K. Vilonen, and T. Xue, Springer correspondence for the split symmetric pair in type A, Compos. Math. 154 (2018), no. 11, 2403-2425.

[CLP] C. De Concini, G. Lusztig, and C. Procesi, Homology of the zero-set of a nilpotent vector field on a flag manifold, J. Amer. Math. Soc. 1 (1988), no. 1, 15-34.

[EM] S. Evens and I. Mirković, Characteristic cycles for the loop Grassmannian and nilpotent orbits, Duke Math. J. 97 (1999), no. 1, 109-126.

[GR] G. Gasper and M. Rahman, Basic Hypergeometric Series, second edition, Encyclopedia of Mathematics and its Applications 96, Cambridge University Press, Cambridge, (2004).

[He] W. H. Hesselink, Desingularizations of varieties of nullforms, Invent. Math. 55 (1979), no. 2, 141163.

[KS] M. Kashiwara and P. Schapira, Sheaves on Manifolds, Grundlehren der Mathematischen Wissenschaften 292, Springer-Verlag, Berlin, (1990).

[KaS] N. M. Katz and P. Sarnak, Random Matrices, Frobenius Eigenvalues, and Monodromy, American Mathematical Society Colloquium Publications 45, American Mathematical Society, Providence, RI, (1999).

$[\mathrm{KR}] \quad$ B. Kostant and S. Rallis, Orbits and representations associated with symmetric spaces, Amer. J. Math. 93 (1971), 753-809.

[L1] G. Lusztig, Intersection cohomology complexes on a reductive group, Invent. Math. 75 (1984), no. 2, 205-272.

[L2] G. Lusztig, Irreducible representations of finite classical groups, Invent. Math. 43 (1977), no. 2, 125-175. 
[L3] G. Lusztig, An induction theorem for Springer's representations, in: Representation Theory of Algebraic Groups and Quantum Groups, pp. 253-259, Adv. Stud. Pure Math. 40, Math. Soc. Japan, Tokyo, (2004).

[R] M. Reeder, Desingularizations of some unstable orbit closures, Pacific J. Math. 167 (1995), no. 2, $327-343$.

[Re] M. Reid, The complete intersection of two or more quadrics, PhD Thesis, Trinity College, Cambridge. (1972).

[S] J. Sekiguchi, The nilpotent subvariety of the vector space associated to a symmetric pair, Publ. Res. Inst. Math. Sci. 20 (1984), no. 1, 155-212.

Department of Mathematics, University of Chicago, Chicago, 60637, USA

Email address: chenth@math.uchicago.edu

School of Mathematics and Statistics, University of Melbourne, Australia and Department of Mathematics and Statistics, University of Helsinki, Finland

Email address: kari.vilonen@unimelb.edu.au

School of Mathematics and Statistics, University of Melbourne, Australia and Department of Mathematics and Statistics, University of Helsinki, Finland

Email address: ting.xue@unimelb.edu.au 